\title{
MULTISCALE DEFORMATION ANALYSIS BY CAUCHY-NAVIER WAVELETS
}

\author{
M. K. ABEYRATNE, W. FREEDEN, AND C. MAYER
}

Received 6 June 2003 and in revised form 20 August 2003

A geoscientifically relevant wavelet approach is established for the classical (inner) displacement problem corresponding to a regular surface (such as sphere, ellipsoid, and actual earth surface). Basic tools are the limit and jump relations of (linear) elastostatics. Scaling functions and wavelets are formulated within the framework of the vectorial CauchyNavier equation. Based on appropriate numerical integration rules, a pyramid scheme is developed providing fast wavelet transform (FWT). Finally, multiscale deformation analysis is investigated numerically for the case of a spherical boundary.

\section{Introduction}

First we recapitulate some results known from the theory of elasticity. We will always regard the inner space $\Sigma_{\text {int }}$ of a closed surface $\Sigma$ as a fixed reference configuration of a body. By a deformation of $\overline{\Sigma_{\text {int }}}$ we mean a oneto-one $c^{1}$-function $z: \overline{\Sigma_{\text {int }}} \rightarrow \mathbb{R}^{3}$ such that $\operatorname{det}(\nabla \otimes z)>0$. The function $u: \overline{\Sigma_{\text {int }}} \rightarrow \mathbb{R}^{3}$, defined by $u(x)=z(x)-x, x \in \overline{\Sigma_{\text {int }}}$, is called the displacement of $\overline{\sum_{\text {int }}}$ relative to the deformation $z$. The tensor field $(\nabla \otimes u)(x)$ is called the displacement gradient. The (infinitesimal) strain tensor is defined by $\mathbf{e}=(1 / 2)\left((\nabla \otimes u)+(\nabla \otimes u)^{T}\right)$ as the symmetric part of the displacement gradient, while the antisymmetric part is used to define the (infinitesimal) rotation tensor $\mathbf{d}$ as $\mathbf{d}=(1 / 2)\left((\nabla \otimes u)-(\nabla \otimes u)^{T}\right)$. While $\mathbf{d}$ describes a "rigid" displacement field, e is responsible for the "nonrigid" displacements. According to Kirchhoff's theorem (see, e.g., [21]) if two displacement fields $u$ and $u^{\prime}$ correspond to the same strain field, then $u=u^{\prime}+w$,

Copyright $\odot 2003$ Hindawi Publishing Corporation

Journal of Applied Mathematics 2003:12 (2003) 605-645

2000 Mathematics Subject Classification: 74B05, 65T60, 86A30, 47H50

URL: http:/ /dx.doi.org/10.1155/S1110757X03206033 
where $w$ is a rigid displacement field. One calls trace $(\mathbf{e})=\nabla \cdot u$ the (elastic) dilatation. Thus the dilatations are determined by the diagonal elements of $\mathbf{e}$, the remaining elements of e prescribe torsions. Every displacement field can be decomposed into a pure torsion (i.e., $\nabla \cdot u=0$ ) and a pure dilatation (i.e., $\nabla \wedge u=0$ ).

An elastic body in a strained configuration performs by definition a tendency of recovering its original form: this tendency is materialized by a field of forces on each part of the body by the other parts. This field of internal forces, called (elastic) stress, is due to the interaction of the molecules of the body which have been removed from their relative position of equilibrium and to recovering this position, following the principle of action and interaction. If $x$ is a point of a (regular) surface element in $\Sigma_{\text {int }}$ with unit normal $v$, then the stress vector $s_{v}(x)=T_{v}(u)(x)$ is the force per unit area at $x$ exerted by the portion of $\Sigma_{\text {int }}$ on the side of the surface element in $\Sigma_{\text {int }}$ towards $v(x)$ on the portion of $\Sigma_{\text {int }}$ on the other side. For time-independent behavior and in the absence of body stress fields, there exists a symmetric tensor field $\mathbf{s}$, called stress tensor field, such that $s_{v}=\mathbf{s} v$ for each vector $v$ and $\nabla(\mathbf{s} a)=0$ for each fixed $a \in$ $\mathbb{R}^{3}$ (for more details see, e.g., $[12,22]$ ).

Hooke's law relates the stress to strain, that is, linear elasticity of the body implies that for each $x \in \Sigma_{\text {int }}$ there exists a linear transformation $\mathrm{C}$ from the space of all tensors into the space of all symmetric tensors such that $\mathbf{s}=\mathrm{Ce}$. The linear theory of elasticity is based on the straindisplacement relation

$$
\mathbf{e}=\frac{1}{2}\left((\nabla \otimes u)+(\nabla \otimes u)^{T}\right)
$$

the stress-strain relation

$$
\mathbf{s}=\mathbf{C e}
$$

and the equation of equilibrium

$$
\operatorname{div} \mathbf{s}+b=0,
$$

where $b$ is the body force field in $\Sigma_{\text {int. }}$ Equations (1.1), (1.2), and (1.3) imply the displacement equation of equilibrium in $\Sigma_{\text {int }}$

$$
\operatorname{div} \mathbf{C}(\nabla \otimes u)+b=0 .
$$

For given $\mathbf{C}$ and $b$, this is a coupled linear system of partial differential equations for the fields $u, \mathbf{e}$, and $\mathbf{s}$. If the material is isotropic, $\mathbf{C}$ is given 
by

$$
\mathbf{C e}=2 \mu \mathbf{e}+\lambda(\text { trace } \mathbf{e}) \mathbf{i},
$$

where the scalars $\lambda$ and $\mu$ are called the Lamé moduli. Moreover, if the material is homogeneous, $\lambda$ and $\mu$ are constants (typical requirements imposed on $\lambda$ and $\mu$ are $\mu>0,3 \lambda+2 \mu>0$ (see, e.g., [16])). Therefore, in the homogeneous isotropic case, observing the identities

$$
\begin{gathered}
\nabla \cdot(\mu(\nabla \otimes u))=\mu \Delta u, \quad \nabla \cdot\left(\mu(\nabla \otimes u)^{T}\right)=0, \\
\nabla \cdot(\lambda(\nabla \cdot u) \mathbf{i})=\lambda \nabla(\nabla \cdot u),
\end{gathered}
$$

we are led to the displacement equation of equilibrium in the form

$$
\mu \Delta u+(\lambda+\mu) \nabla \nabla u+b=0 .
$$

Finally, assuming that the body force field $b$ vanishes, this equation can be reduced to the so-called Cauchy-Navier equation in $\Sigma_{\text {int }}$

$$
\mu \Delta u+(\lambda+\mu) \nabla \nabla u=0 .
$$

This equation plays the same part in the theory of elasticity as the Laplace equation in the theory of harmonic functions and it formally reduces to it for $\mu=1$ and $\lambda=-1$. The Cauchy-Navier equation allows an equivalent formulation in $\Sigma_{\text {int }}$

$$
\Delta u+\sigma \nabla \nabla \cdot u=0
$$

where $\sigma=(1-2 \rho)^{-1}, \rho=\lambda / 2(\lambda+\mu), \mu \neq 0$. The quantity $\rho$ is the Poisson ratio. For simplicity, we let

$$
\diamond u=\mu \Delta u+(\lambda+\mu) \nabla \nabla u=0
$$

in $\Sigma_{\text {int }}$. It is easy to show that the displacement field $u$ is biharmonic and its divergence and curl are harmonic. This yields a deep relation between linear elasticity and potential theory (see, e.g., [17]).

The layout of this report on multiscale deformation analysis by Cauchy-Navier wavelets is as follows: after a brief sketch of the theory of linear elasticity given in the introduction (Section 1), we deal with some preliminary concepts of elastic potentials in Section 2. In analogy to the classical potential theoretic case we discuss (in Section 3) the limit 
and jump relations within the framework of the Hilbert space of squareintegrable vector fields on a regular surface $\Sigma$. The uniqueness, existence, and regularity of the solution of the displacement boundary value problem of elastostatics are discussed in Section 4. Next (in Section 5) a wavelet approach is introduced based on the layer potentials and their operator formulation in the nomenclature of the Hilbert space of squareintegrable vector fields on the regular boundary $\Sigma$. We introduce the socalled (Cauchy-Navier) $\Sigma$-scaling functions and wavelets. The wavelet transform and the reconstruction formulae both in continuous and discrete formulations are explicitly written down. The geomathematically relevant (inner) three-dimensional displacement boundary value problem of elastostatics is treated within the multiscale structure of CauchyNavier wavelets. Finally, Section 6 is devoted to numerical applications of wavelet approximation on the sphere.

\section{Preliminaries}

We begin by introducing some preliminaries that will be used throughout this paper.

\subsection{Notation}

As usual, $\mathbb{R}^{3}$ denotes the three-dimensional (real) Euclidean space. We consistently write $x, y, \ldots$ for the elements of $\mathbb{R}^{3}$. In components, we have the representation $x=x_{1} \epsilon^{1}+x_{2} \epsilon^{2}+x_{3} \epsilon^{3}$, where the vectors $\epsilon^{1}, \epsilon^{2}$, and $\epsilon^{3}$ form the canonical orthonormal basis in $\mathbb{R}^{3}$. The inner product, vector product, and tensor product between $x$ and $y$, respectively, are defined as usual by

$$
\begin{gathered}
x \cdot y=x^{T} y=\sum_{i=1}^{3} x_{i} y_{i} \\
x \wedge y=\left(x_{2} y_{3}-x_{3} y_{2}, x_{3} y_{1}-x_{1} y_{3}, x_{1} y_{3}-y_{3} y_{1}\right)^{T}, \\
x \otimes y=x y^{T}=\left(\begin{array}{lll}
x_{1} y_{1} & x_{1} y_{2} & x_{1} y_{3} \\
x_{2} y_{1} & x_{2} y_{2} & x_{2} y_{3} \\
x_{3} y_{1} & x_{3} y_{2} & x_{3} y_{3}
\end{array}\right) .
\end{gathered}
$$

Furthermore, the Euclidean norm of $x$ is denoted by $|x|$, that is, $|x|=$ $(x \cdot x)^{1 / 2}$. The unit sphere in $\mathbb{R}^{3}$ is denoted by $\Omega$. More explicitly,

$$
\Omega=\left\{\xi \in \mathbb{R}^{3}|| \xi \mid=1\right\}
$$


M. K. Abeyratne et al. 609

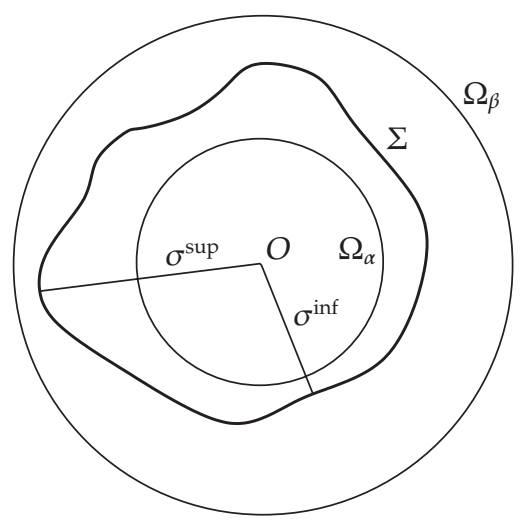

FIGURE 2.1. Configuration of a regular surface.

\subsection{Regular surfaces}

A surface $\Sigma \subset \mathbb{R}^{3}$ is called regular if it satisfies the following properties:

(i) $\Sigma$ divides $\mathbb{R}^{3}$ uniquely into the bounded region $\Sigma_{\text {int }}$ (inner space) and the unbounded region $\Sigma_{\text {ext }}$ (outer space) given by $\Sigma_{\text {ext }}=\mathbb{R}^{3} \backslash$ $\overline{\sum_{\text {int }}}, \overline{\sum_{\text {int }}}=\sum_{\text {int }} \cup \Sigma$,

(ii) $\Sigma$ is a closed and compact surface free of double points,

(iii) $\sum_{\text {int }}$ contains the origin,

(iv) $\sum$ is locally of class $C^{(2)}$.

Given a regular surface (see Figure 2.1), then there exist positive constants $\alpha$ and $\beta$ such that

$$
\alpha<\sigma^{\mathrm{inf}}=\inf _{x \in \Sigma}|x| \leq \sup _{x \in \Sigma}|x|=\sigma^{\text {sup }}<\beta
$$

The sets $\Omega_{\alpha}$ and $\Omega_{\beta}$ denote the spheres of radii $\alpha$ and $\beta$, respectively. As usual, $\Omega_{\beta}^{\text {int }}$ and $\Omega_{\beta}^{\text {ext }}$ (resp., $\Omega_{\alpha}^{\text {int }}$ and $\Omega_{\alpha}^{\text {ext }}$ ) denote the inner and outer spaces of $\Omega_{\beta}$ (resp., $\Omega_{\alpha}$ ).

A vector field $f$ possessing $k$ continuous derivatives is said to be of class $c^{(k)}, 0 \leq k \leq \infty$. The space $c^{(0)}(\Sigma)(=c(\Sigma))$ is the class of continuous vector fields $f$ defined on $\Sigma$. The space $c(\Sigma)$ is a complete normed space endowed with the norm $\|f\|_{c(\Sigma)}=\sup _{x \in \Sigma}|f(x)|$. In $c(\Sigma)$ we have the inner product $(\cdot, \cdot)_{\ell^{2}(\Sigma)}$ corresponding to the norm

$$
\|f\|_{\ell^{2}(\Sigma)}=\left(\int_{\Sigma}|f(x)|^{2} d \omega(x)\right)^{1 / 2}
$$


where $d \omega$ represents the surface element on $\Sigma$. Furthermore, for each $f \in c(\Sigma)$, we have the norm estimate

$$
\|f\|_{\ell^{2}(\Sigma)} \leq\|\Sigma\|\|f\|_{c(\Sigma)}, \quad\|\Sigma\|=\left(\int_{\Sigma} d \omega\right)^{1 / 2}
$$

By $\ell^{2}(\Sigma)$ we denote the space of (Lebesgue) square-integrable vector fields on $\Sigma$. It is a Hilbert space with respect to the inner product $(\cdot, \cdot)_{\ell^{2}(\Sigma)}$ and a Banach space with respect to $\|\cdot\|_{\ell^{2}(\Sigma)}$. The space $\ell^{2}(\Sigma)$ is the completion of $c(\Sigma)$ with respect to the norm $\|\cdot\|_{\ell^{2}(\Sigma)}$, that is,

$$
\ell^{2}(\Sigma)=\overline{c(\Sigma)}\|\cdot\|_{\ell^{2}(\Sigma)}
$$

By $\operatorname{pot}\left(\Sigma_{\text {int }}\right)$ we denote the space of potentials $u \in c^{(2)}(\Sigma)$ satisfying the Cauchy-Navier equation $\diamond u=\mu \Delta u+(\lambda+\mu) \nabla \nabla u=0$ in $\Sigma_{\text {int }}$ (with $\lambda$ and $\mu$ being fixed). With pot $\left(\overline{\Sigma_{\text {int }}}\right)$ we denote the space of all vector fields $u: \overline{\Sigma_{\text {int }}} \rightarrow \mathbb{R}^{3}$ satisfying the properties

(1) $u \in c^{(2)}\left(\Sigma_{\text {int }}\right) \cap c\left(\overline{\Sigma_{\text {int }}}\right)$,

(2) $\left.u\right|_{\Sigma_{\text {int }}} \in \operatorname{pot}\left(\Sigma_{\text {int }}\right)$.

\section{Potential operators}

Elastostatics may be formulated by a vector potential theory which closely parallels classical scalar potential theory. As a matter of fact, the displacement vector corresponds to the scalar harmonic function, whereas the traction vector corresponds to the normal derivative. Well-known integral formulae parallel the Gauss flux theorem, Betti's and Somigliana's formulae parallel Green's formulae. Moreover, vector potentials may be constructed in close orientation to the scalar single- and double-layer potentials. The resulting boundary integral equations show analogous properties to the scalar boundary integral equations. As a consequence, the fundamental existence-uniqueness theorems of classical elastostatics can be formulated in analogy to the corresponding theorems of harmonic function theory. For more details, the reader is referred to [16], which gives the theoretical treatment of the vector theory. Further theoretical aspects can be found in many books; for example, $[12,15,20]$.

At each point $x$ of a regular surface $\Sigma$ we can construct a normal $v(x)$ pointing into the outer space $\Sigma_{\text {ext }}$. The set

$$
\Sigma(\tau)=\left\{x_{\tau} \in \mathbb{R}^{3} \mid x_{\tau}=x+\tau \mathcal{v}(x), x \in \Sigma\right\}
$$


generates a parallel surface which is exterior for $\tau>0$ and interior for $\tau<$ 0 . It is known that, if $|\tau|$ is sufficiently small, the parallel surface is regular and the normal to one parallel surface is normal to the other.

The matrix $\Gamma(x), x \in \mathbb{R}^{3}$ with $|x| \neq 0$, given by

$$
\Gamma(x)=\frac{\lambda+3 \mu}{2 \mu(\lambda+2 \mu)}\left(\left(\epsilon^{i} \cdot \epsilon^{k}+\frac{(\lambda+\mu)}{\lambda+3 \mu} \frac{\left(x \cdot \epsilon^{i}\right)\left(x \cdot \epsilon^{k}\right)}{|x|^{2}}\right) \frac{1}{|x|}\right)_{i, k=1,2,3}
$$

is constituted by the so-called fundamental solutions $\Gamma_{k}(x)=\Gamma(x) \epsilon^{k}, k=$ $1,2,3$, associated to the operator $\diamond$ (cf. [17]).

The operator

$$
N=\frac{1}{\lambda+3 \mu}\left(2 \mu(\lambda+2 \mu) \frac{\partial}{\partial \mathcal{v}}+(\lambda+\mu)(\lambda+2 \mu) \mathcal{v} \operatorname{div}+\mu(\lambda+\mu) \mathcal{v} \times \operatorname{curl}\right)
$$

is called the (pseudo-)stress operator. Furthermore, $N_{x} \Gamma_{k}(x), x \in \mathbb{R}^{3}$ with $|x| \neq 0$, is given by

$$
N_{x} \Gamma_{k}(x)=\left(\frac{\partial}{\partial v(x)} \frac{1}{|x|}\right) \Lambda_{k}(x)
$$

where

$$
\Lambda_{k}(x)=\frac{2 \mu}{\lambda+3 \mu}\left(\epsilon^{k}+\frac{3(\lambda+\mu)}{2 \mu} \frac{\left(\epsilon^{k} \cdot x\right) x}{|x|^{2}}\right), \quad k=1,2,3
$$

We let

$$
\Lambda(x)=\left(\Lambda_{i}(x) \cdot \epsilon^{k}\right)_{i, k^{\prime}} \quad i, k=1,2,3
$$

Limit and jump relations by layer potentials such as single-layer potential, double-layer potential, and $N$-derivative of the single-layer potential (which parallels the normal derivative in classical potential theory) are known from the literature. For example, [17] gave their formulation in pointwise sense for a regular surface. The papers [3,9] introduced an adequate operator notation to establish the validity of the limit and jump relations in uniform sense (i.e., in the topology of $\left.\|\cdot\|_{C(\Sigma)}\right)$.

In what follows, we adopt this operator notation to give a generalization of the limit and jump relations in the topology of $\|\cdot\|_{\ell^{2}(\Sigma)}$. The generalization will be given exclusively by functional analytic means (based on the results in uniform metric). Later on, the $\ell^{2}(\Sigma)$-formulations of the limit and jump relations are the essential tools for introducing vector 
612 Multiscale deformation analysis by Cauchy-Navier wavelets

wavelets (associated to the Cauchy-Navier operator) on regular surfaces within $\ell^{2}(\Sigma)$-topology.

Assuming $|\tau|$ to be sufficiently small, we define the so-called potential operators $P(\tau), P_{N}(\tau)$, and $N_{P}(\tau)$, respectively, by the following integrals:

$$
P(\tau) g(x)=\int_{\Sigma} \Gamma\left(x_{\tau}-y\right) g(y) d \omega(y)
$$

$P(\tau)$ is the operator of the single-layer potential on $\Sigma$ for values on $\Sigma(\tau)$,

$$
P_{N}(\tau) g(x)=\int_{\Sigma}\left(\frac{\partial}{\partial \mathcal{v}(y)} \frac{1}{\left|x_{\tau}-y\right|}\right) \Lambda\left(x_{\tau}-y\right) g(y) d \omega(y),
$$

$P_{N}(\tau)$ is the operator of the double-layer potential on $\Sigma$ for values on $\Sigma(\tau)$,

$$
\begin{aligned}
N_{P}(\tau) g(x) & =\int_{\Sigma}\left(\frac{\partial}{\partial v(x)} \frac{1}{\left|x_{\tau}-y\right|}\right) \Lambda\left(x_{\tau}-y\right) g(y) d \omega(y) \\
& =N_{x} \int_{\Sigma} \Gamma\left(x_{\tau}-y\right) g(y) d \omega(y)
\end{aligned}
$$

$N_{P}(\tau)$ is the $N$-derivative of the single-layer potential on $\Sigma$ for values on $\Sigma(\tau)$.

The operators $P(\tau), P_{N}(\tau)$, and $N_{P}(\tau)$ form mappings from $\ell^{2}(\Sigma)$ into $c(\Sigma)$ provided that $|\tau|$ is sufficiently small. Furthermore, the integrals formally defined by

$$
\begin{gathered}
P(0) g(x)=\int_{\Sigma} \Gamma(x-y) g(y) d \omega(y), \\
P_{N}(0) g(x)=\int_{\Sigma}\left(\frac{\partial}{\partial v(y)} \frac{1}{|x-y|}\right) \Lambda(x-y) g(y) d \omega(y), \\
N_{P}(0) g(x)=\int_{\Sigma}\left(\frac{\partial}{\partial v(x)} \frac{1}{|x-y|}\right) \Lambda(x-y) g(y) d \omega(y)
\end{gathered}
$$

exist and define linear bounded operators $P(0), P_{N}(0)$, and $N_{P}(0)$ mapping $\ell^{2}(\Sigma)$ into $c(\Sigma)$.

As usual, the dual operator $P^{*}(\tau)$ of the operator $P(\tau)$ is defined by

$$
\int_{\Sigma} g(x) \cdot P(\tau) f(x) d \omega(x)=\int_{\Sigma} P^{*}(\tau) g(x) \cdot f(x) d \omega(x)
$$


for $f, g \in \ell^{2}(\Sigma)$. In connection with the property $\Gamma(x)=\Gamma(-x), x \in \Sigma$, this shows that

$$
P^{*}(\tau) g(x)=\int_{\Sigma} \Gamma\left(x-y_{\tau}\right) g(y) d \omega(y)
$$

In the same way we obtain

$$
\begin{aligned}
& P_{N}^{*}(\tau) g(x)=\int_{\Sigma}\left(\frac{\partial}{\partial \mathcal{v}(x)} \frac{1}{\left|x-y_{\tau}\right|}\right) \Lambda\left(x-y_{\tau}\right) g(y) d \omega(y), \\
& N_{P}^{*}(\tau) g(x)=\int_{\Sigma}\left(\frac{\partial}{\partial \mathcal{v}(y)} \frac{1}{\left|x-y_{\tau}\right|}\right) \Lambda\left(x-y_{\tau}\right) g(y) d \omega(y) .
\end{aligned}
$$

As mentioned before, the potential operators in elastostatics (see, e.g., [17]) behave near the boundary much like the ordinary harmonic potential operators. In particular, limit formulae and jump relations can be formulated in close orientation to the potential theoretic case. To be more explicit, let $I$ be the identity operator in $\ell^{2}(\Sigma)$. For all $\tau>0$ sufficiently small, the operators $L_{i}^{ \pm}(\tau), i=1,2,3$, and $J_{i}(\tau), i=1,2,3,4,5$, are defined by

$$
\begin{gathered}
L_{1}^{ \pm}(\tau)=P( \pm \tau)-P(0), \\
L_{2}^{ \pm}(\tau)=P_{N}( \pm \tau)-P_{N}(0) \mp 2 \pi I \\
L_{3}^{ \pm}(\tau)=N_{P}( \pm \tau)-N_{P}(0) \pm 2 \pi I \\
J_{1}(\tau)=P(\tau)-P(-\tau), \\
J_{2}(\tau)=P_{N}(\tau)-P_{N}(-\tau)-4 \pi I, \\
J_{3}(\tau)=N_{P}(\tau)-N_{P}(-\tau)+4 \pi I, \\
J_{4}(\tau)=P_{N}(\tau)+P_{N}(-\tau)-2 P_{N}(0), \\
J_{5}(\tau)=N_{P}(\tau)+N_{P}(-\tau)-2 N_{P}(0),
\end{gathered}
$$

respectively. Then, for all $g \in c(\Sigma)$,

$$
\begin{aligned}
& \lim _{\substack{\tau \rightarrow 0 \\
\tau>0}}\left\|L_{i}^{ \pm}(\tau) g\right\|_{c(\Sigma)}=0, \quad i=1,2,3, \\
& \lim _{\substack{\tau \rightarrow 0 \\
\tau>0}}\left\|J_{i}(\tau) g\right\|_{c(\Sigma)}=0, \quad i=1,2,3,4,5 .
\end{aligned}
$$

In addition, the adjoint operators with respect to the inner product $(\cdot, \cdot)_{\ell^{2}(\Sigma)}$ are bounded linear operators with respect to the norm $\|\cdot\|_{c(\Sigma)}$ (see, e.g., $[3,17]$ ). 
614 Multiscale deformation analysis by Cauchy-Navier wavelets

Theorem 3.1. For all $g \in \ell^{2}(\Sigma)$,

$$
\begin{aligned}
& \lim _{\substack{\tau \rightarrow 0 \\
\tau>0}}\left\|L_{i}^{ \pm}(\tau) g\right\|_{\ell^{2}(\Sigma)}=0, \quad i=1,2,3, \\
& \lim _{\substack{\tau \rightarrow 0 \\
\tau>0}}\left\|J_{i}(\tau) g\right\|_{\ell^{2}(\Sigma)}=0, \quad i=1,2,3,4,5 .
\end{aligned}
$$

The proof of Theorem 3.1 can be found in the appendix.

\section{Uniqueness, existence, and regularity}

In the notations given above, the homogeneous isotropic elastic displacement boundary value problem can be formulated as follows. Given $f \in$ $c(\Sigma)$, find a vector field $u \in \operatorname{pot}\left(\overline{\bar{\Sigma}_{\text {int }}}\right)$ satisfying the boundary condition $\left.u\right|_{\Sigma}=f$. As it is well-known, the boundary value problem has a unique solution (see, e.g., [16]). In order to prove the existence, we use the double-layer potential

$$
\begin{aligned}
u(x) & =P_{N}(0) g(x) \\
& =\int_{\Sigma}\left(\frac{\partial}{\partial v(y)} \frac{1}{|x-y|}\right) \Lambda(x-y) g(y) d \omega(y), \quad g \in c(\Sigma) .
\end{aligned}
$$

Observing the discontinuity of the double-layer potential, we obtain from (3.19) that

$$
f(x)=-2 \pi g(x)+\int_{\Sigma}\left(\frac{\partial}{\partial v(y)} \frac{1}{|x-y|}\right) \Lambda(x-y) g(y) d \omega(y)
$$

for all $x \in \Sigma$. The resulting integral equation $-f=\left(2 \pi I-P_{N}(0)\right) g, g \in$ $c(\Sigma)$, fulfills all standard Fredholm theorems.

The homogeneous integral equation $\left(2 \pi I-P_{N}(0)\right) g=0$ has no solution different from $g=0$. Thus, the solution of the boundary value problem exists and is representable by a double-layer potential as indicated in (4.1). For details, the reader is referred to [17]. The operator $T=2 \pi I-P_{N}(0)$ and its adjoint operator $T^{*}$ (with respect to the scalar product $\left.(\cdot, \cdot)_{\ell^{2}(\Sigma)}\right)$ form mappings from $c(\Sigma)$ into $c(\Sigma)$ which are linear and bounded with respect to the norm $\|\cdot\|_{c(\Sigma)}$. The operators $T$ and $T^{*}$ in $c(\Sigma)$ are injective and, by the Fredholm alternative, bijective in the Banach space $c(\Sigma)$. Consequently, by the open mapping theorem (see [23]), the operators $T^{-1}, T^{*-1}$ are linear and bounded with respect to $\|\cdot\|_{c(\Sigma)}$. Moreover, $\left(T^{*}\right)^{-1}=\left(T^{-1}\right)^{*}$. But this implies that both $T^{-1}$ and $\left(T^{*}\right)^{-1}$ are 
bounded with respect to the norm $\|\cdot\|_{\ell^{2}(\Sigma)}$ in $c(\Sigma)$. As we have shown, for a given $f \in c(\Sigma)$, there exists a vector field $g \in c(\Sigma)$ determined by (4.2) such that $u$ is representable in the form (4.1). Suppose that $\mathcal{K}$ is a subset of $\Sigma_{\text {int }}$ with $\operatorname{dist}(\mathcal{K}, \Sigma)>0$. Then Cauchy-Schwarz inequality applied to (4.1) gives, for each $x \in \mathcal{K}$,

$$
\begin{aligned}
|u(x)| \leq & \left(\int_{\Sigma} \sum_{k=1}^{3}\left|\left(\frac{\partial}{\partial v(y)} \frac{1}{|x-y|}\right) \Lambda_{k}(x-y)\right|^{2} d \omega(y)\right)^{1 / 2} \\
& \times\left(\int_{\Sigma}|g(y)|^{2} d \omega(y)\right)^{1 / 2} .
\end{aligned}
$$

But this means that

$$
\sup _{x \in \mathcal{K}}|u(x)| \leq E\|g\|_{\ell^{2}(\Sigma)}
$$

where

$$
E=\sup _{x \in \mathcal{K}}\left(\int_{\Sigma} \sum_{k=1}^{3}\left|\left(\frac{\partial}{\partial v(y)} \frac{1}{|x-y|}\right) \Lambda_{k}(x-y)\right|^{2} d \omega(y)\right)^{1 / 2} .
$$

In connection with (4.2) this implies the existence of a positive constant $B$ (depending on $\Sigma$ and $\mathcal{K}$ ) such that

$$
\sup _{x \in \mathcal{K}}|u(x)| \leq E\left\|T^{-1} f\right\|_{\ell^{2}(\Sigma)} \leq B\|f\|_{\ell^{2}(\Sigma)} .
$$

Summarizing our results, we obtain the following regularity condition.

THEOREM 4.1. Let $u$ be a vector field of class $\operatorname{pot}\left(\overline{\Sigma_{\text {int }}}\right)$ and $\mathcal{K}$ a subset of $\Sigma_{\text {int }}$ with $\operatorname{dist}(\mathcal{K}, \Sigma)>0$. Then

$$
\sup _{x \in \mathcal{K}}|u(x)| \leq B\left(\int_{\Sigma}|u(x)|^{2} d \omega(x)\right)^{1 / 2} .
$$

In other words, $l^{2}(\Sigma)$-approximation implies approximation in $\Sigma_{\text {int }}$ in locally compact topology.

\section{Cauchy-Navier wavelets}

In Sections 3 and 4, we used the operator nomenclature of layer potentials to extend the limit and jump relations to the $\|\cdot\|_{\ell^{2}(\Sigma)}$-topology and 
616 Multiscale deformation analysis by Cauchy-Navier wavelets

to discuss the well-posedness of the inner displacement problem. In this section, we go back to the integral representations of the layer potentials. Completely written out in the $l^{2}(\Sigma)$-framework, Theorem 3.1 then reads as follows.

THEOREM 5.1. For $f \in \ell^{2}(\Sigma)$,

$$
\begin{aligned}
& \lim _{\substack{\tau \rightarrow 0 \\
\tau>0}}\left(\int_{\Sigma} \mid \int_{\Sigma} \Phi_{\tau}^{(1)}(x, y) f(y) d \omega(y)\right. \\
& \left.-\left.\int_{\Sigma} \Gamma(x-y) f(y) d \omega(y)\right|^{2} d \omega(x)\right)^{1 / 2}=0, \\
& \lim _{\substack{\tau \rightarrow 0 \\
\tau>0}}\left(\int_{\Sigma}\left|\int_{\Sigma} \Phi_{\tau}^{(2)}(x, y) f(y) d \omega(y)-f(x)\right|^{2} d \omega(x)\right)^{1 / 2}=0, \\
& \lim _{\substack{\tau \rightarrow 0 \\
\tau>0}}\left(\int_{\Sigma}\left|\int_{\Sigma} \Phi_{\tau}^{(3)}(x, y) f(y) d \omega(y)-f(x)\right|^{2} d \omega(x)\right)^{1 / 2}=0, \\
& \lim _{\substack{\tau \rightarrow 0 \\
\tau>0}}\left(\int_{\Sigma}\left|\int_{\Sigma} \Phi_{\tau}^{(4)}(x, y) f(y) d \omega(y)-0\right|^{2} d \omega(x)\right)^{1 / 2}=0, \\
& \lim _{\substack{\tau \rightarrow 0 \\
\tau>0}}\left(\int_{\Sigma}\left|\int_{\Sigma} \Phi_{\tau}^{(5)}(x, y) f(y) d \omega(y)-f(x)\right|^{2} d \omega(x)\right)^{1 / 2}=0, \\
& \lim _{\substack{\tau \rightarrow 0 \\
\tau>0}}\left(\int_{\Sigma}\left|\int_{\Sigma} \Phi_{\tau}^{(6)}(x, y) f(y) d \omega(y)-f(x)\right|^{2} d \omega(x)\right)^{1 / 2}=0, \\
& \lim _{\substack{\tau \rightarrow 0 \\
\tau>0}}\left(\int_{\Sigma} \mid \int_{\Sigma} \Phi_{\tau}^{(7)}(x, y) f(y) d \omega(y)\right. \\
& \left.-\left.\int_{\Sigma}\left(\frac{\partial}{\partial \mathcal{v}(y)} \frac{1}{|x-y|}\right) \Lambda(x-y) f(y) d \omega(y)\right|^{2} d \omega(x)\right)^{1 / 2}=0, \\
& \lim _{\substack{\tau \rightarrow 0 \\
\tau>0}}\left(\int_{\Sigma} \mid \int_{\Sigma} \Phi_{\tau}^{(8)}(x, y) f(y) d \omega(y)\right. \\
& \left.-\left.\int_{\Sigma}\left(\frac{\partial}{\partial v(x)} \frac{1}{|x-y|}\right) \Lambda(x-y) f(y) d \omega(y)\right|^{2} d \omega(x)\right)^{1 / 2}=0,
\end{aligned}
$$


where

$$
\begin{aligned}
& \boldsymbol{\Phi}_{\tau}^{(1)}(x, y)=\Gamma\left(x_{\tau}-y\right), \\
& \mathbf{\Phi}_{\tau}^{(2)}(x, y)=\frac{1}{2 \pi}\left(\left(\frac{\partial}{\partial \mathcal{v}(y)} \frac{1}{\left|x_{\tau}-y\right|}\right) \Lambda\left(x_{\tau}-y\right)\right. \\
& \left.-\left(\frac{\partial}{\partial \mathcal{v}(y)} \frac{1}{|x-y|}\right) \Lambda(x-y)\right) \\
& \mathbf{\Phi}_{\tau}^{(3)}(x, y)=-\frac{1}{2 \pi}\left(\left(\frac{\partial}{\partial \mathcal{v}(x)} \frac{1}{\left|x_{\tau}-y\right|}\right) \Lambda\left(x_{\tau}-y\right)\right. \\
& \left.-\left(\frac{\partial}{\partial \mathcal{v}(x)} \frac{1}{|x-y|}\right) \Lambda(x-y)\right) \\
& \boldsymbol{\Phi}_{\tau}^{(4)}(x, y)=\Gamma\left(x_{\tau}-y\right)-\Gamma\left(x_{-\tau}-y\right), \\
& \boldsymbol{\Phi}_{\tau}^{(5)}(x, y)=\frac{1}{4 \pi}\left(\left(\frac{\partial}{\partial \mathcal{v}(y)} \frac{1}{\left|x_{\tau}-y\right|}\right) \Lambda\left(x_{\tau}-y\right)\right. \\
& \left.-\left(\frac{\partial}{\partial v(y)} \frac{1}{\left|x_{-\tau}-y\right|}\right) \Lambda\left(x_{-\tau}-y\right)\right), \\
& \mathbf{\Phi}_{\tau}^{(6)}(x, y)=-\frac{1}{4 \pi}\left(\left(\frac{\partial}{\partial \mathcal{v}(x)} \frac{1}{\left|x_{\tau}-y\right|}\right) \Lambda\left(x_{\tau}-y\right)\right. \\
& \left.-\left(\frac{\partial}{\partial \mathcal{v}(x)} \frac{1}{\left|x_{-\tau}-y\right|}\right) \Lambda\left(x_{-\tau}-y\right)\right), \\
& \mathbf{\Phi}_{\tau}^{(7)}(x, y)=\frac{1}{2}\left(\left(\frac{\partial}{\partial \mathcal{v}(y)} \frac{1}{\left|x_{\tau}-y\right|}\right) \Lambda\left(x_{\tau}-y\right)\right. \\
& \left.+\left(\frac{\partial}{\partial v(y)} \frac{1}{\left|x_{-\tau}-y\right|}\right) \Lambda\left(x_{-\tau}-y\right)\right), \\
& \mathbf{\Phi}_{\tau}^{(8)}(x, y)=\frac{1}{2}\left(\left(\frac{\partial}{\partial \mathcal{v}(x)} \frac{1}{\left|x_{\tau}-y\right|}\right) \Lambda\left(x_{\tau}-y\right)\right. \\
& \left.+\left(\frac{\partial}{\partial \mathcal{v}(x)} \frac{1}{\left|x_{-\tau}-y\right|}\right) \Lambda\left(x_{-\tau}-y\right)\right),
\end{aligned}
$$

$\tau>0,(x, y) \in \Sigma \times \Sigma$.

Briefly formulated, we obtain the following corollary. 
618 Multiscale deformation analysis by Cauchy-Navier wavelets

Corollary 5.2. For $f \in \ell^{2}(\Sigma)$,

$$
\begin{aligned}
\lim _{\substack{\tau \rightarrow 0 \\
\tau>0}} \int_{\Sigma} \boldsymbol{\Phi}_{\tau}^{(i)}(x, y) f(y) d \omega(y) & \\
= & \begin{cases}\int_{\Sigma} \Gamma(x-y) f(y) d \omega(y), & i=1, \\
0, & i=4, \\
f(x), & i=2,3,5,6, \\
\int_{\Sigma}\left(\frac{\partial}{\partial \mathcal{v}(y)} \frac{1}{|x-y|}\right) \Lambda(x-y) f(y) d \omega(y), & i=7, \\
\int_{\Sigma}\left(\frac{\partial}{\partial v(x)} \frac{1}{|x-y|}\right) \Lambda(x-y) f(y) d \omega(y), & i=8,\end{cases}
\end{aligned}
$$

holds in the sense of the $\|\cdot\|_{\ell^{2}(\Sigma)}$-norm.

Figure 5.1 gives graphical illustrations of matrix norm, diagonal, and nondiagonal components of $\boldsymbol{\Phi}_{\tau}^{(5)}(\cdot, \cdot)$.

\subsection{Scaling and wavelet functions}

For $\tau>0$ and $i=1, \ldots, 8$, the family $\left\{\boldsymbol{\Phi}_{\tau}^{(i)}\right\}_{\tau>0}$ of kernels $\boldsymbol{\Phi}_{\tau}^{(i)}: \Sigma \times \Sigma \rightarrow \mathbb{R}^{3 \times 3}$ is called a (Cauchy-Navier) $\Sigma$-scaling function of type $i$. Moreover, $\boldsymbol{\Phi}_{1}^{(i)}$ : $\Sigma \times \Sigma \rightarrow \mathbb{R}^{3 \times 3}$ (i.e., with $\tau=1$ ) is called the mother kernel of the (CauchyNavier) $\Sigma$-scaling function of type $i$. Correspondingly, for $\tau>0$ and $i=$ $1, \ldots, 8$, the family $\left\{\boldsymbol{\Psi}_{\tau}^{(i)}\right\}_{\tau>0}$ of kernels $\boldsymbol{\Psi}_{\tau}^{(i)}: \Sigma \times \Sigma \rightarrow \mathbb{R}^{3 \times 3}$ given by

$$
\Psi_{\tau}^{(i)}(x, y)=-(\alpha(\tau))^{-1} \frac{d}{d \tau} \boldsymbol{\Phi}_{\tau}^{(i)}(x, y), \quad x, y \in \Sigma,
$$

is called a (Cauchy-Navier) $\sum$-wavelet function of type $i$. Moreover, $\Psi_{1}^{(i)}$ : $\Sigma \times \Sigma \rightarrow \mathbb{R}^{3 \times 3}$ defines the so-called mother kernel of the (Cauchy-Navier) $\Sigma$-wavelet of type $i$. It should be noted that (5.4) is called the (scale continuous) $\Sigma$-scaling equation. The factor $\alpha(\tau)^{-1}$ can be chosen in an appropriate way. For simplicity, throughout the remainder of this paper, we will use $\alpha(\tau)=\tau^{-1}$.

Definition 5.3. Let $\left\{\boldsymbol{\Phi}_{\tau}^{(i)}\right\}_{\tau>0}$ be a $\Sigma$-scaling function of type $i$. Then the associated $\Sigma$-wavelet transform of type $i(\mathrm{WT})^{(i)}: \ell^{2}(\Sigma) \rightarrow \ell^{2}((0, \infty), \Sigma)$ of a function $f \in \ell^{2}(\Sigma)$ is defined by

$$
(\mathrm{WT})^{(i)}(f)(\tau, x)=\int_{\Sigma} \Psi_{\tau}^{(i)}(x, y) f(y) d \omega(y) .
$$




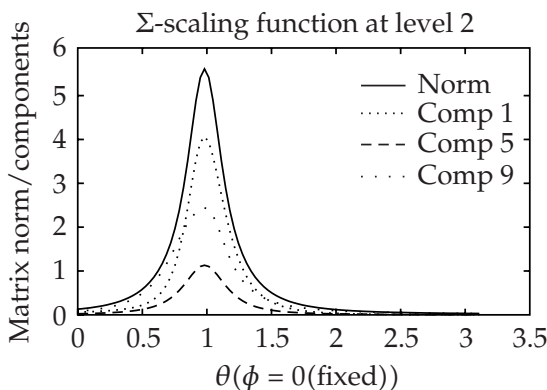

(a) Diagonal/norm.

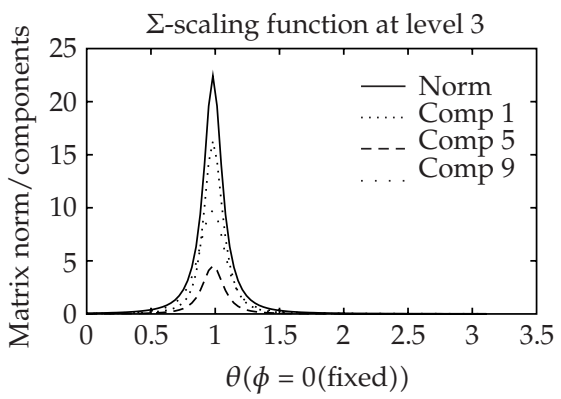

(c) Diagonal/norm.

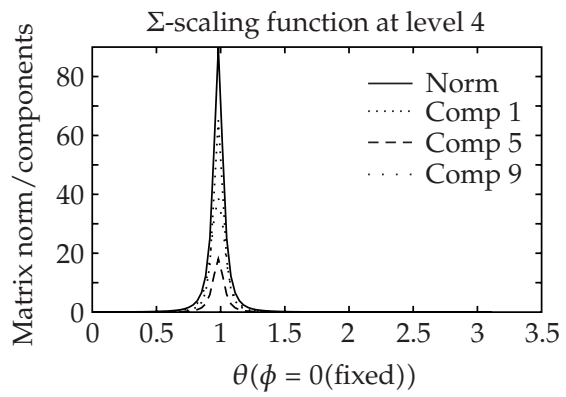

(e) Diagonal/norm.

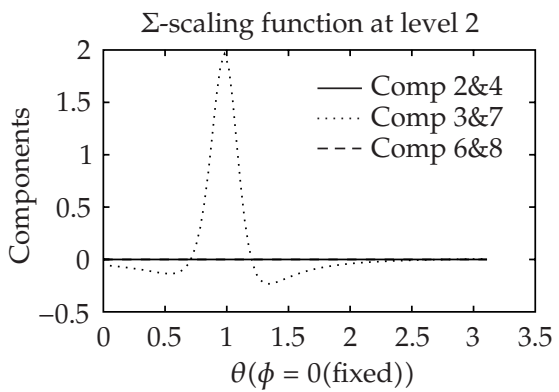

(b) Nondiagonal.

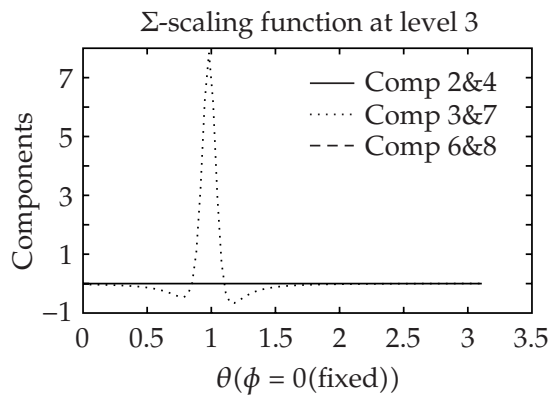

(d) Nondiagonal.

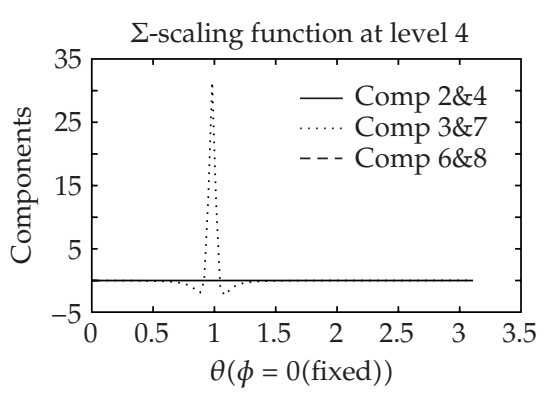

(f) Nondiagonal.

FIGURE 5.1. Matrix norm (Frobenius norm), diagonal, and nondiagonal components of the $\Sigma$-scaling function $\boldsymbol{\Phi}_{\tau}^{(5)}(\cdot, \cdot)$ for $\tau=2^{-j}$, $j=2,3,4$. 
620 Multiscale deformation analysis by Cauchy-Navier wavelets

\subsection{Scale continuous reconstruction formula}

It is not difficult to show that the $\sum$-wavelet functions $\boldsymbol{\Psi}_{\tau}^{(i)}, i=1, \ldots, 8$, behave (componentwise) like $O\left(\tau^{-1}\right)$, hence the convergence of the integrals occurring in the next theorem is guaranteed.

THEOREM 5.4. Let $\left\{\boldsymbol{\Phi}_{\tau}^{(i)}\right\}_{\tau>0}$ be a $\sum$-scaling function of type $i$. Suppose that $f$ is of class $\ell^{2}(\Sigma)$. Then the reconstruction formula

$$
\begin{aligned}
\int_{0}^{\infty}(\mathrm{WT})^{(i)}(f)(\tau, x) \frac{d \tau}{\tau} & \left(\begin{array}{ll}
\int_{\Sigma} \Gamma(x-y) f(y) d \omega(y), & i=1 \\
0, & i=4 \\
f(x), & i=2,3,5,6 \\
\int_{\Sigma}\left(\frac{\partial}{\partial \mathcal{v}(y)} \frac{1}{|x-y|}\right) \Lambda(x-y) f(y) d \omega(y), & i=7 \\
\int_{\Sigma}\left(\frac{\partial}{\partial \mathcal{v}(x)} \frac{1}{|x-y|}\right) \Lambda(x-y) f(y) d \omega(y), & i=8
\end{array}\right.
\end{aligned}
$$

holds in the sense of $\|\cdot\|_{\ell^{2}(\Sigma)}$.

Proof. Let $R>0$ be arbitrary. Taking from (5.4) the identity

$$
\boldsymbol{\Phi}_{R}^{(i)}(x, y)=\int_{R}^{\infty} \Psi_{\tau}^{(i)}(x, y) \frac{d \tau}{\tau}, \quad x, y \in \Sigma,
$$

we obtain

$$
\begin{aligned}
\int_{R}^{\infty}(\mathrm{WT})^{(i)}(f)(\tau, x) \frac{d \tau}{\tau} & =\int_{R}^{\infty} \int_{\Sigma} \Psi_{\tau}^{(i)}(x, y) f(y) d \omega(y) \frac{d \tau}{\tau} \\
& =\int_{\Sigma}\left(\int_{R}^{\infty} \Psi_{\tau}^{(i)}(x, y) \frac{d \tau}{\tau}\right) f(y) d \omega(y) \\
& =\int_{\Sigma} \boldsymbol{\Phi}_{R}^{(i)}(x, y) f(y) d \omega(y) .
\end{aligned}
$$

Letting $R$ tend to 0 , we get the desired result from Theorem 5.1. 
Next, we are interested in formulating the wavelet transform and the reconstruction formula by using the so-called "shift" and "dilation" operators. We define the $x$-shift and $\tau$-dilation of a mother kernel, respectively, by

$$
\begin{gathered}
T_{x}: \boldsymbol{\Phi}_{1}^{(i)} \longmapsto T_{x} \boldsymbol{\Phi}_{1}^{(i)}=\boldsymbol{\Phi}_{1, x}^{(i)}(\cdot)=\boldsymbol{\Phi}_{1}^{(i)}(x, \cdot), \quad x \in \Sigma, \\
D_{\tau}: \boldsymbol{\Phi}_{1}^{(i)} \longmapsto D_{\tau} \boldsymbol{\Phi}_{1}^{(i)}=\boldsymbol{\Phi}_{\tau}^{(i)}, \quad \tau>0 .
\end{gathered}
$$

Consequently, we obtain by composition

$$
T_{x} D_{\tau} \mathbf{\Phi}_{1}^{(i)}=T_{x} \mathbf{\Phi}_{\tau}^{(i)}=\mathbf{\Phi}_{\tau, x}^{(i)}(\cdot)=\mathbf{\Phi}_{\tau}^{(i)}(x, \cdot), \quad i=1, \ldots, 8 .
$$

Analogously,

$$
T_{x} D_{\tau} \boldsymbol{\Psi}_{1}^{(i)}=T_{x} \boldsymbol{\Psi}_{\tau}^{(i)}=\boldsymbol{\Psi}_{\tau, x}^{(i)}(\cdot)=\boldsymbol{\Psi}_{\tau}^{(i)}(x, \cdot), \quad i=1, \ldots, 8
$$

We can thus state the following theorem.

THEOREM 5.5. For $x \in \sum$ and $f \in \ell^{2}(\Sigma)$,

$$
\begin{aligned}
& \lim _{\substack{\tau \rightarrow 0 \\
\tau>0}} \int_{\Sigma} T_{x} D_{\tau} \boldsymbol{\Phi}_{1}^{(i)}(y) f(y) d \omega(y) \\
& = \begin{cases}\int_{\Sigma} \Gamma(x-y) f(y) d \omega(y), & i=1, \\
0, & i=4, \\
f(x), & i=2,3,5,6, \\
\int_{\Sigma}\left(\frac{\partial}{\partial v(y)} \frac{1}{|x-y|}\right) \Lambda(x-y) f(y) d \omega(y), & i=7, \\
\int_{\Sigma}\left(\frac{\partial}{\partial v(x)} \frac{1}{|x-y|}\right) \Lambda(x-y) f(y) d \omega(y), & i=8,\end{cases} \\
& \int_{0}^{\infty} \int_{\Sigma} T_{x} D_{\tau} \Psi_{1}^{(i)}(y) f(y) d \omega(y) \frac{d \tau}{\tau} \\
& = \begin{cases}\int_{\Sigma} \Gamma(x-y) f(y) d \omega(y), & i=1, \\
0, & i=4, \\
f(x), & i=2,3,5,6, \\
\int_{\Sigma}\left(\frac{\partial}{\partial v(y)} \frac{1}{|x-y|}\right) \Lambda(x-y) f(y) d \omega(y), & i=7, \\
\int_{\Sigma}\left(\frac{\partial}{\partial v(x)} \frac{1}{|x-y|}\right) \Lambda(x-y) f(y) d \omega(y), & i=8,\end{cases}
\end{aligned}
$$

hold in the sense of $\|\cdot\|_{\ell^{2}(\Sigma)}$. 
622 Multiscale deformation analysis by Cauchy-Navier wavelets

In other words, the wavelets are used as mathematical means for breaking up a complicated structure of an $\ell^{2}(\Sigma)$-vector field $(i=2,3,5,6)$ or a vector potential $(i=1,7,8)$ into simple pieces at different scales and positions.

\subsection{Scale discrete reconstruction formula}

Until now emphasis has been put on the whole scale interval $(0, \infty)$. Next, discretization of the wavelet transform will be discussed in the form of "wavelet packets."

Let $\left(\tau_{j}\right)_{j \in \mathbb{Z}}$ denote a (monotonically decreasing) sequence of numbers satisfying the properties

$$
\lim _{\tau \rightarrow \infty} \tau_{j}=0, \quad \lim _{\tau \rightarrow-\infty} \tau_{j}=\infty,
$$

(e.g., $\tau_{j}=2^{-j}, j \in \mathbb{Z}$ ). Given a $\sum$-scaling function $\left\{\boldsymbol{\Phi}_{\tau}^{(i)}\right\}_{\tau>0}$ of type $i$, we define the (scale) discretized $\Sigma$-scaling function of type $i$ by $\left\{\boldsymbol{\Phi}_{\tau_{j}}^{(i)}\right\}_{j \in \mathbb{Z}}$. Then we are obviously led by Theorem 5.5 to the following result.

THEOREM 5.6. For $f \in \ell^{2}(\Sigma)$, the limit

$$
\begin{aligned}
\lim _{j \rightarrow \infty} \int_{\Sigma} T_{x} D_{\tau_{j}} \mathbf{\Phi}_{1}^{(i)}(y) f(y) d \omega(y) & \\
= & \begin{cases}\int_{\Sigma} \Gamma(x-y) f(y) d \omega(y), & i=1, \\
0, & i=4, \\
f(x), & i=2,3,5,6, \\
\int_{\Sigma}\left(\frac{\partial}{\partial v(y)} \frac{1}{|x-y|}\right) \Lambda(x-y) f(y) d \omega(y), & i=7, \\
\int_{\Sigma}\left(\frac{\partial}{\partial v(x)} \frac{1}{|x-y|}\right) \Lambda(x-y) f(y) d \omega(y), & i=8\end{cases}
\end{aligned}
$$

holds in the $\|\cdot\|_{\ell^{2}(\Sigma)}$-sense.

Definition 5.7. Let $\left\{\boldsymbol{\Phi}_{\tau_{j}}^{(i)}\right\}_{j \in \mathbb{Z}}$ be a discretized $\Sigma$-scaling function of type $i$. Then the (scale) discretized $\Sigma$-wavelet (packet) function of type $i$ is defined by

$$
\Psi_{\tau_{j}}^{(i)}(x, y)=\int_{\tau_{j+1}}^{\tau_{j}} \Psi_{\tau}^{(i)}(x, y) \frac{d \tau}{\tau}, \quad j \in \mathbb{Z}, x, y \in \Sigma, i=1, \ldots, 8
$$


With the definition of $\Psi_{\tau_{j}}^{(i)}$ as given by (5.15) we immediately obtain that

$$
\Psi_{\tau_{j}}^{(i)}(x, y)=-\int_{\tau_{j+1}}^{\tau_{j}} \tau \frac{d}{d \tau} \boldsymbol{\Phi}_{\tau}^{(i)}(x, y) \frac{d \tau}{\tau}=\boldsymbol{\Phi}_{\tau_{j+1}}^{(i)}-\boldsymbol{\Phi}_{\tau_{j}}^{(i)}, \quad x, y \in \Sigma
$$

Equation (5.16) is called the (scale) discretized $\sum$-scaling equation of type $i$. It should be remarked that, with a suitably chosen $\tau_{j}$, formula (5.16) can easily be used to formulate the $\Sigma$-wavelet function in a discrete form once the $\sum$-scaling function has been given. To be more specific, assume that $f$ is a vector field of class $\ell^{2}(\Sigma)$ and consider the discretized $\Sigma$-scaling equation of type $i$. Then, for $J \in \mathbb{Z}, N \in \mathbb{N}$, and $f \in \ell^{2}(\Sigma)$, we have

$$
\begin{aligned}
\int_{\Sigma} \boldsymbol{\Phi}_{\tau_{J+1}}^{(i)}(x, y) f(y) d \omega(y) & \\
= & \int_{\Sigma} \boldsymbol{\Phi}_{\tau_{J}}^{(i)}(x, y) f(y) d \omega(y) \\
& +\int_{\Sigma} \Psi_{\tau_{J}}^{(i)}(x, y) f(y) d \omega(y), \quad x \in \Sigma .
\end{aligned}
$$

Therefore, the $J$ th partial wavelet reconstruction of $f \in \ell^{2}(\Sigma)$ may be understood as the difference between two "smoothings" at consecutive scales, $J+1$ and $J$. Observing the property of a telescoping sum, we see that

$$
\begin{aligned}
\int_{\Sigma} \boldsymbol{\Phi}_{\tau_{J+N}}^{(i)}(x, y) f(y) d \omega(y) & \\
= & \int_{\Sigma} \boldsymbol{\Phi}_{\tau_{J}}^{(i)}(x, y) f(y) d \omega(y) \\
& +\sum_{j=J}^{J+N-1} \int_{\Sigma} \Psi_{\tau_{j}}^{(i)}(x, y) f(y) d \omega(y), \quad x \in \Sigma .
\end{aligned}
$$

By taking into account the property (5.18) together with Theorem 5.5, we find the following theorem. 
624 Multiscale deformation analysis by Cauchy-Navier wavelets

THEOREM 5.8. Let $\left\{\boldsymbol{\Phi}_{\tau_{j}}^{(i)}\right\}_{j \in \mathbb{Z}}$ be a (scale) discretized $\Sigma$-scaling function of type $i$. Then the multiscale representation of a function $f \in \ell^{2}(\Sigma)$

$$
\begin{aligned}
\int_{\Sigma} \mathbf{\Phi}_{\tau_{J}}^{(i)}(x, y) f(y) d \omega(y)+\sum_{j=J}^{\infty} \int_{\Sigma} \Psi_{\tau_{j}}^{(i)}(x, y) f(y) d \omega(y) \\
\quad= \begin{cases}\int_{\Sigma} \Gamma(x-y) f(y) d \omega(y), & i=1, \\
0, & i=4, \\
f(x), & i=2,3,5,6, \\
\int_{\Sigma}\left(\frac{\partial}{\partial v(y)} \frac{1}{|x-y|}\right) \Lambda(x-y) f(y) d \omega(y), & i=7, \\
\int_{\Sigma}\left(\frac{\partial}{\partial v(x)} \frac{1}{|x-y|}\right) \Lambda(x-y) f(y) d \omega(y), & i=8,\end{cases}
\end{aligned}
$$

holds for all $J \in \mathbb{Z}$ in the sense of $\|\cdot\|_{\ell^{2}(\Sigma)}$.

Using the so-called (scale) discretized $\Sigma$-wavelet transform of type $i$ canonically given by

$$
(\mathrm{WT})^{i}(f)\left(\tau_{j} ; x\right)=\int_{\Sigma} \Psi_{\tau_{j} ; x}^{(i)}(y) f(y) d \omega(y), \quad x \in \Sigma,
$$

we are able to derive the following corollary.

Corollary 5.9. Let $\left\{\boldsymbol{\Phi}_{\tau_{j}}^{(i)}\right\}_{j \in \mathbb{Z}}$ be a (scale) discretized $\Sigma$-scaling function of type $i$. Then, for all $f \in \ell^{2}(\Sigma)$,

$$
\begin{aligned}
& \sum_{j=-\infty}^{\infty}(\mathrm{WT})^{i}(f)\left(\tau_{j} ; x\right) \\
& \quad= \begin{cases}\int_{\Sigma} \Gamma(x-y) f(y) d \omega(y), & i=1, \\
0, & i=4, \\
f(x), & i=2,3,5,6, \\
\int_{\Sigma}\left(\frac{\partial}{\partial v(y)} \frac{1}{|x-y|}\right) \Lambda(x-y) f(y) d \omega(y), & i=7, \\
\int_{\Sigma}\left(\frac{\partial}{\partial v(x)} \frac{1}{|x-y|}\right) \Lambda(x-y) f(y) d \omega(y), & i=8,\end{cases}
\end{aligned}
$$

holds in the $\|\cdot\|_{\ell^{2}(\Sigma)}$-sense. 


\subsection{Scale and detail spaces}

As in the spherical theory of wavelets (see $[6,7,10]$ for more details), the operators $P_{\tau_{j}}^{(i)}$ and $R_{\tau_{j}}^{(i)}$ defined by

$$
\begin{aligned}
& P_{\tau_{j}}^{(i)}=\int_{\Sigma} \boldsymbol{\Phi}_{\tau_{j}}^{(i)}(\cdot, y) f(y) d \omega(y), \quad f \in \ell^{2}(\Sigma), \\
& R_{\tau_{j}}^{(i)}=\int_{\Sigma} \Psi_{\tau_{j}}^{(i)}(\cdot, y) f(y) d \omega(y), \quad f \in \ell^{2}(\Sigma),
\end{aligned}
$$

may be understood as a lowpass filter and a bandpass filter, respectively. The scale spaces $V_{\tau_{j}}^{(i)}$ and details spaces $W_{\tau_{j}}^{(i)}$ of type $i$ are defined by

$$
\begin{aligned}
V_{\tau_{j}}^{(i)} & =\left\{P_{\tau_{j}}^{(i)}(f) \mid f \in \ell^{2}(\Sigma)\right\}, \\
W_{\tau_{j}}^{(i)} & =\left\{R_{\tau_{j}}^{(i)}(f) \mid f \in \ell^{2}(\Sigma)\right\},
\end{aligned}
$$

respectively. It is clear that

$$
P_{\tau_{J+1}}^{(i)}(f)=P_{\tau_{J}}^{(i)}(f)+R_{\tau_{J}}^{(i)}, \quad J \in \mathbb{Z}
$$

Consequently,

$$
V_{\tau_{J+1}}^{(i)}=V_{\tau_{J}}^{(i)}+W_{\tau_{J}}^{(i)}
$$

Furthermore,

$$
V_{\tau_{J+1}}^{(i)}=V_{\tau_{J_{0}}}^{(i)}+\sum_{j=0}^{J} W_{\tau_{j}}^{(i)}
$$

It should be remarked that the sum (5.27), in general, is neither direct nor orthogonal. Furthermore,

$$
\begin{aligned}
& \overline{\bigcup_{j=-\infty}^{\infty} V_{\tau_{j}}^{(i)}\|\cdot\|_{\ell^{2}(\Sigma)}}=\ell^{2}(\Sigma), \quad i=2,3,5,6, \\
& \overline{\bigcup_{j=-\infty}^{\infty} V_{\tau_{j}}^{(1)}\|\|_{\ell^{2}(\Sigma)}}=P(0)\left(\ell^{2}(\Sigma)\right),
\end{aligned}
$$


626 Multiscale deformation analysis by Cauchy-Navier wavelets

$$
\begin{aligned}
& \overline{\bigcup_{j=-\infty}^{\infty} V_{\tau_{j}}^{(7)}} \|_{\ell^{2}(\Sigma)}=P_{N}(0)\left(\ell^{2}(\Sigma)\right), \\
& \overline{\bigcup_{j=-\infty}^{\infty} V_{\tau_{j}}^{(8)}} \|^{\cdot \|_{\ell^{2}(\Sigma)}}=N_{P}(0)\left(\ell^{2}(\Sigma)\right) .
\end{aligned}
$$

The following scheme briefly summarizes the essential steps of our wavelet approach (for $i=2,3,5,6$ ):

$$
\begin{gathered}
P_{\tau_{0}}^{(i)} f+R_{\tau_{0}}^{(i)} f+\cdots+R_{\tau_{J}}^{(i)} f+\cdots=f, \\
V_{\tau_{0}}^{(i)}+W_{\tau_{0}}^{(i)}+\cdots+W_{\tau_{J}}^{(i)}+\cdots=\ell^{2}(\Sigma),
\end{gathered}
$$

where $P_{\tau_{0}}^{(i)} f \in V_{\tau_{0}}^{(i)}, R_{\tau_{0}}^{(i)} f \in W_{\tau_{0}}^{(i)}, \ldots, R_{\tau_{J}}^{(i)} f \in W_{\tau_{J}}^{(i)}, \ldots, f \in \ell^{2}(\Sigma)$.

\subsection{A tree algorithm}

In what follows, we present a particular scheme which utilizes the computational process of the reconstruction and decomposition of the wavelet approximation. This is known as tree algorithm that provides a recursive process to compute the integrals $P_{\tau_{j}}^{(i)}(f)$ and $R_{\tau_{j}}^{(i)}(f)$ on different levels, starting from an initial approximation of a given $f \in \ell^{2}(\Sigma)$ without falling back upon the original vector field $f$ in each step.

Let $\left\{\left(y_{k}^{N_{j}}, w_{k}^{N_{j}}\right)\right\}$ constitute an appropriate integration rule on $\Sigma$ with given nodes $y_{k}^{N_{j}} \in \Sigma$ and weights $w_{k}^{N_{j}} \in \mathbb{R}$. Assume that for sufficiently large $J \in \mathbb{N}$ there exist coefficient vectors $a_{k}^{N_{J}} \in \mathbb{R}^{3}, k=1, \ldots, N_{J}$, such that

$$
P_{\tau_{J}}^{(i)}(f)(x)=\sum_{k=1}^{N_{J}} \mathbf{\Phi}_{\tau_{J}}^{(i)}\left(x, y_{k}^{N_{J}}\right) a_{k}^{N_{J}}, \quad i=1, \ldots, 8, x \in \Sigma .
$$

Now we want to introduce an algorithm to obtain the coefficients $a^{N_{j}}=$ $\left(a_{1}^{N_{j}}, \ldots, a_{N_{j}}^{N_{j}}\right) \in \mathbb{R}^{3} \times \mathbb{R}^{N_{j}}, j=J_{0}, \ldots, J$, such that

(a) the vector $a^{N_{j}}$ is obtainable from $a^{N_{j+1}}, j=J_{0}, \ldots, J-1$, 
(b) the expressions $P_{\tau_{j}}^{(i)}(f)(x)$ and $R_{\tau_{j-1}}^{(i)}(f)(x)$ can be written as

$$
\begin{aligned}
P_{\tau_{j}}^{(i)}(f)(x) & =\sum_{k=1}^{N_{j}} \boldsymbol{\Phi}_{\tau_{j}}^{(i)}\left(x, y_{k}^{N_{j}}\right) a_{k}^{N_{j}}, \quad j=J_{0}, \ldots, J, \\
R_{\tau_{j-1}}^{(i)}(f)(x) & =\sum_{k=1}^{N_{j-1}} \Psi_{\tau_{j-1}}^{(i)}\left(x, y_{k}^{N_{j}}\right) a_{k}^{N_{j-1}}, \quad j=J_{0}+1, \ldots, J .
\end{aligned}
$$

For this scheme, we use appropriately chosen approximate integration rules such that $P_{\tau_{j}}^{(i)}(f)$ and $R_{\tau_{j}}^{(i)}(f)$ can be represented by

$$
\begin{aligned}
P_{\tau_{j}}^{(i)}(f)(x) & \approx \sum_{k=1}^{N_{j}} w_{k}^{N_{j}} \boldsymbol{\Phi}_{\tau_{j}}^{(i)}\left(x, y_{k}^{N_{j}}\right) f\left(y_{k}^{N_{j}}\right), \\
R_{\tau_{j-1}}^{(i)}(f)(x) & \approx \sum_{k=1}^{N_{j-1}} w_{k}^{N_{j-1}} \Psi_{\tau_{j-1}}^{(i)}\left(x, y_{k}^{N_{j}}\right) f\left(y_{k}^{N_{j-1}}\right),
\end{aligned}
$$

where $\left\{\left(y_{k}^{N_{j}}, w_{k}^{N_{j}}\right) \in \Sigma \times \mathbb{R}\right\}$ are the prescribed integration points and nodes and " $\approx$ " means that the remainder is negligibly small.

The tree algorithm can be divided into two parts, namely the initial step and the pyramid step. For the initial step we assume as indicated by (5.31) that $J \in \mathbb{N}$ is sufficiently large. Thus we are able to start with

$$
a_{k}^{N_{J}} \simeq w_{k}^{N_{J}} f\left(y_{k}^{N_{J}}\right), \quad k=1, \ldots, N_{J}
$$

The aim of the pyramid step is to construct $a^{N_{j}}$ from $a^{N_{j+1}}$ by recursion. At this point, it is essential to assume that there exist (tensor) kernel functions $\Xi_{j}^{(i)}: \Sigma \times \Sigma \rightarrow \mathbb{R}^{3 \times 3}$ such that

$$
\begin{aligned}
& \boldsymbol{\Phi}_{\tau_{j}}^{(i)}(x, y) \approx \int_{\Sigma} \Phi_{\tau_{j}}^{(i)}(x, z) \Xi_{j}^{(i)}(z, y) d \omega(z), \\
& \Xi_{j}^{(i)}(x, y) \approx \int_{\Sigma} \Xi_{j}^{(i)}(x, z) \Xi_{j+1}^{(i)}(z, y) d \omega(z),
\end{aligned}
$$

for $j=J_{0}, \ldots, J$. A reasonable choice for $\Xi_{j}^{(i)}$ is

$$
\Xi_{j}^{(i)}=\boldsymbol{\Phi}_{\tau_{J+L}}^{(i)}, \quad j=J_{0}, \ldots, J ; i \in\{2,3,5,6\},
$$


628 Multiscale deformation analysis by Cauchy-Navier wavelets

with $L \in \mathbb{N}$ suitably large. By the aforementioned approximate integration rules we obtain

$$
\begin{aligned}
\int_{\Sigma} \boldsymbol{\Phi}_{\tau_{j}}^{(i)}(x, y) f(y) d \omega(y) & \approx \int_{\Sigma}\left[\int_{\Sigma} \boldsymbol{\Phi}_{\tau_{j}}^{(i)}(x, z) \Xi_{j}^{(i)}(z, y) d \omega(z)\right] f(y) d \omega(y) \\
& =\int_{\Sigma} \boldsymbol{\Phi}_{\tau_{j}}^{(i)}(x, z)\left[\int_{\Sigma} \Xi_{j}^{(i)}(z, y) f(y) d \omega(y)\right] d \omega(z) \\
& \approx \sum_{k=1}^{N_{j}} \boldsymbol{\Phi}_{\tau_{j}}^{(i)}\left(x, y_{k}^{N_{j}}\right) a_{k}^{N_{j}}
\end{aligned}
$$

where

$$
a_{k}^{N_{j}}=w_{k}^{N_{j}} \int_{\Sigma} \Xi_{j}^{(i)}\left(y_{k}^{N_{j}}, y\right) f(y) d \omega(y), \quad j=J_{0}, \ldots, J-1
$$

Hence, in connection with (5.37), we find

$$
\begin{aligned}
a_{k}^{N_{j}} & =w_{k}^{N_{j}} \int_{\Sigma} \Xi_{j}^{(i)}\left(y_{k}^{N_{j}}, y\right) f(y) d \omega(y) \\
& \approx w_{k}^{N_{j}} \int_{\Sigma}\left[\int_{\Sigma} \Xi_{j}^{(i)}\left(y_{k}^{N_{j}}, x\right) \Xi_{j+1}^{(i)}(x, y) d \omega(y)\right] f(y) d \omega(y) \\
& \approx w_{k}^{N_{j}} \int_{\Sigma} \sum_{l=1}^{N_{j+1}} \Xi_{j}^{(i)}\left(y_{k}^{N_{j}}, y_{l}^{N_{j+1}}\right) \Xi_{j+1}^{(i)}\left(y_{l}^{N_{j+1}}, y\right) f(y) d \omega(y) \\
& =w_{k}^{N_{j}} \sum_{l=1}^{N_{j+1}} \Xi_{j}^{(i)}\left(y_{k}^{N_{j}}, y_{l}^{N_{j+1}}\right) a_{l}^{N_{j+1}}
\end{aligned}
$$

for $j=J-1, \ldots, J_{0}$ and $k=1, \ldots, N_{j}$.

In conclusion, once the coefficients $a^{N_{j}}$ are calculated, the coefficients $a^{N_{j-1}}$ are obtained by (5.39). Starting from an initial value $a^{N_{J}}$, all the coefficient vectors can be calculated recursively. Note that the coefficients $a^{N_{J}}$ in the initial step do not depend on the choice of $\Xi_{J}^{(i)}=\boldsymbol{\Phi}_{\tau_{J+L}}^{(i)}$. Furthermore, the functions $\Xi_{j}^{(i)}, j=J_{0}, \ldots, J$, can be chosen independently of the $\Sigma$-scaling function used in the integrals $P_{\tau_{j}}^{(i)}(f)$ and $R_{\tau_{j}}^{(i)}(f)$. 
Finally, with the proposed tree algorithm prescribed by (5.34) and (5.39), the decomposition and reconstruction process of the wavelet approximation can be illustrated briefly as follows:

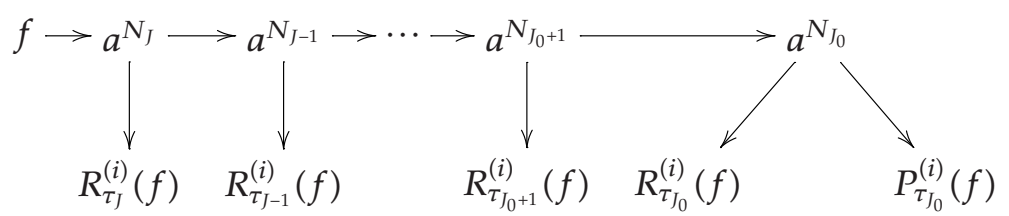

(decomposition scheme),

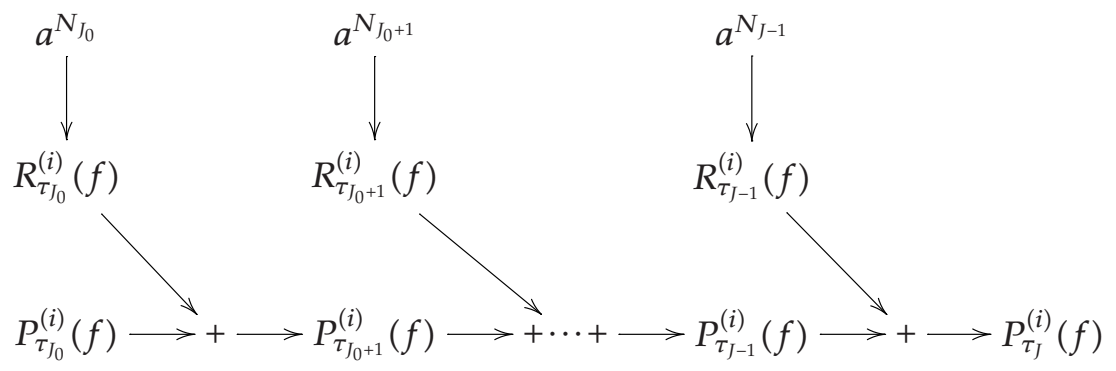

(reconstruction scheme).

5.6. Multiscale solution of the (inner) displacement boundary value problem

Next, we discuss the solution of the (inner) displacement boundary value problem by means of the wavelet approximation techniques (derived in preceding sections). The existence, uniqueness, and regularity of the solutions of such problems are known from Section 4.

For given $f \in c(\Sigma)$, the solution $u \in \operatorname{pot}\left(\overline{\Sigma_{\text {int }}}\right)$ with $\left.u\right|_{\Sigma}=f$ of the (inner) displacement problem can be expressed uniquely by a double-layer potential

$$
u(x)=\int_{\Sigma}\left(\frac{\partial}{\partial v(y)} \frac{1}{|x-y|}\right) \Lambda(x-y) g(y) d \omega(y), \quad g \in c(\Sigma)
$$

The corresponding integral equation reads as follows:

$$
2 \pi I-P_{N}(0) g=-f, \quad g \in c(\Sigma)
$$


More explicitly,

$$
\begin{gathered}
2 \pi g(x)-\int_{\Sigma}\left(\frac{\partial}{\partial v(y)} \frac{1}{|x-y|}\right) \Lambda(x-y) g(y) d \omega(y) \\
=-f(x), \quad g \in c(\Sigma), x \in \Sigma .
\end{gathered}
$$

To approximate $u$ given by the double-layer potential in (5.42), we use the concept stated in Theorem 5.1. In accordance with this approach, we are able to rewrite (5.44) approximately in the form

$$
2 \pi g(x)-\int_{\Sigma} \mathbf{\Phi}_{\tau_{L}}^{(7)}(x, y) g(y) d \omega(y)=-f(x), \quad x \in \Sigma,
$$

provided that $L \in \mathbb{N}$ is sufficiently large. Once the boundary integral equation (5.45) is solved, the density function $g$ is inserted into (5.42) and, thereby the (approximate) solution $u$ is obtained in $\Sigma_{\text {int. }}$. Like in many cases of boundary integral equations, there is, in general, no straightforward way of constructing the unknown function $g$. It is, therefore, necessary to apply a suitable approximation method. In this respect, it is canonical for us to go back to Theorem 5.1 that enables us to formulate

$$
g(y)=\lim _{j \rightarrow \infty} \int_{\Sigma} \boldsymbol{\Phi}_{\tau_{j}}^{(5)}(y, z) g(z) d \omega(z)
$$

Using an appropriate numerical integration technique, an approximation of $g$ of level $J$, denoted by $g_{J}$, can be expressed by

$$
g_{J}(y)=\sum_{l=1}^{N_{J}} w_{l}^{N_{J}} \mathbf{\Phi}_{\tau_{J}}^{(5)}\left(y, y_{l}^{N_{J}}\right) g\left(y_{l}^{N_{J}}\right)=\sum_{l=1}^{N_{J}} \mathbf{\Phi}_{\tau_{J}}^{(5)}\left(y, y_{l}^{N_{J}}\right) a_{l}^{N_{J}},
$$

where $w_{l}^{N_{I}}, l=1, \ldots, N_{J}$, are the integration weights corresponding to the nodal points $y_{l}^{N_{J}} \in \Sigma, l=1, \ldots, N_{J}$, and $a_{l}^{N_{J}} \in \mathbb{R}^{3}, l=1, \ldots, N_{J}$.

The unknowns $a_{l}^{N_{J}} \in \mathbb{R}^{3}, l=1, \ldots, N_{J}$, are deducible from (5.45) by solving a system of linear equations obtained by a suitable approximation method such as collocation, Galerkin procedure, least square approximation, and so forth (see, e.g., [5]). In consequence we are led to 
the following system of equations for the unknowns $a_{l}^{N_{J}}, l=1, \ldots, N_{J}$ :

$$
\begin{aligned}
& \sum_{l=1}^{N_{J}}\left(2 \pi \boldsymbol{\Phi}_{\tau_{J}}^{(5)}\left(y_{m}^{N_{J}}, y_{l}^{N_{J}}\right)-\sum_{k=1}^{N_{J}} w_{k}^{N_{J}} \boldsymbol{\Phi}_{\tau_{J}}^{(5)}\left(y_{m}^{N_{J}}, y_{k}^{N_{J}}\right) \boldsymbol{\Phi}_{\tau_{L}}^{(7)}\left(y_{k}^{N_{J}}, y_{l}^{N_{J}}\right)\right) a_{l}^{N_{J}} \\
& =-f\left(y_{m}^{N_{J}}\right), \quad m=1, \ldots, N_{J}
\end{aligned}
$$

However, such a consideration leads to a system of linear equations with a "full" matrix which seems to require much computational work for the definition of the matrix as well as for the solution. In this context, taking into consideration the localization behavior of the kernel functions, suitable accelerating techniques such as panel clustering, domain decomposition, and so forth can efficiently be used (see, e.g., $[11,13,14]$ ). However, further modifications of such techniques relevant to this particular consideration will be needed. This is a challenge for future work.

In this respect, a variant of our tree algorithm comes into play: once the starting values (see (5.34)) $a^{N_{J}}=\left(a_{1}^{N_{J}}, \ldots, a_{N_{J}}^{N_{J}}\right)^{T} \in \mathbb{R}^{3 \times N_{J}}$ are given, the coefficients $a^{N_{j}}=\left(a_{1}^{N_{j}}, \ldots, a_{N_{j}}^{N_{j}}\right)^{T} \in \mathbb{R}^{3 \times N_{j}}, j=J_{0}, \ldots, J-1$, are obtained by the recursion formula

$$
a_{k}^{N_{j}}=w_{k}^{N_{j}} \sum_{l=1}^{N_{j+1}} \Xi_{j}^{(i)}\left(y_{k}^{N_{j}}, y_{l}^{N_{j+1}}\right) a_{l}^{N_{j+1}}, \quad k=1, \ldots, N_{j}
$$

The corresponding approximate integrals are obtained by

$$
\begin{gathered}
P_{\tau_{j}}^{(i)}(g)(x) \approx \sum_{k=1}^{N_{j}} \boldsymbol{\Phi}_{\tau_{j}}^{(5)}\left(x, y_{k}^{N_{j}}\right) a_{k}^{N_{j}}, \quad x \in \Sigma, j=J_{0}, \ldots, J, \\
R_{\tau_{j-1}}^{(i)}(g)(x) \approx \sum_{k=1}^{N_{j}} \Psi_{\tau_{j-1}}^{(5)}\left(x, y_{k}^{N_{j}}\right) a_{k}^{N_{j}}, \quad x \in \Sigma, j=J_{0}+1, \ldots, J,
\end{gathered}
$$

where

$$
R_{\tau_{j-1}}^{(i)}(g)(x)=P_{\tau_{j}}^{(i)}(g)(x)-P_{\tau_{j-1}}^{(i)}(g)(x)
$$

Hence, we finally arrive at the following theorem for the (inner) displacement boundary value problems of the Cauchy-Navier theory.

THEOREM 5.10. Let $\Sigma$ be a regular surface. For given $f \in c(\Sigma)$, let $u$ be the potential of class $\operatorname{pot}\left(\overline{\sum_{\text {int }}}\right)$ with $\left.u\right|_{\Sigma}=f$. Then the function $f_{J} \in c(\Sigma)$ given by 
632 Multiscale deformation analysis by Cauchy-Navier wavelets

$$
\begin{aligned}
f_{J}(x)= & 2 \pi \sum_{l=1}^{N_{J_{0}}} \boldsymbol{\Phi}_{\tau_{J_{0}}}^{(i)}\left(x, y_{l}^{N_{J_{0}}}\right) a_{l}^{N_{J_{0}}}+\sum_{j=J_{0}}^{J-1} 2 \pi \sum_{l=1}^{N_{j}} \boldsymbol{\Psi}_{\tau_{j}}^{(i)}\left(x, y_{l}^{N_{j}}\right) a_{l}^{N_{j}} \\
& -\sum_{l=1}^{N_{J_{0}}}\left(\int_{\Sigma} \boldsymbol{\Phi}_{\tau_{L}}^{(7)}(x, y) \boldsymbol{\Phi}_{\tau_{J_{0}}}^{(i)}\left(y, y_{l}^{N_{J_{0}}}\right) d \omega(y)\right) a_{l}^{N_{J_{0}}} \\
& -\sum_{j=J_{0}}^{J-1} \sum_{l=1}^{N_{j}}\left(\int_{\Sigma} \boldsymbol{\Phi}_{\tau_{L}}^{(7)}(x, y) \boldsymbol{\Psi}_{\tau_{j}}^{(i)}\left(y, y_{l}^{N_{j}}\right) d \omega(y)\right) a_{l}^{N_{j}}
\end{aligned}
$$

$x \in \Sigma$, represents a J-scale approximation of $f \in c(\Sigma)$ in the sense of $\|\cdot\|_{\ell^{2}(\Sigma)}$, where $i=2,3,5,6$ and $L \in \mathbb{N}$ is sufficiently large. Furthermore, by virtue of Theorem 4.1,

$$
\begin{aligned}
u_{J}= & \sum_{l=1}^{N_{J_{0}}} \int_{\Sigma} \boldsymbol{\Phi}_{\tau_{L}}^{(7)}(\cdot, y) \boldsymbol{\Phi}_{\tau_{J_{0}}}^{(i)}\left(y, y_{l}^{N_{J_{0}}}\right) d \omega(y) a_{l}^{N_{J_{0}}} \\
& +\sum_{j=J_{0}}^{J-1} \sum_{l=1}^{N_{J}} \int_{\Sigma} \boldsymbol{\Phi}_{\tau_{L}}^{(7)}(\cdot, y) \Psi_{\tau_{J}}^{(i)}\left(y, y_{l}^{N_{J}}\right) d \omega(y) a_{l}^{N_{j}}
\end{aligned}
$$

represents a $J$-scale approximation of $u$ in the sense of $\|\cdot\|_{c(\mathcal{K})}$ for every $\mathcal{K} \subset$ $\Sigma_{\text {int }}$ with $\operatorname{dist}(\overline{\mathcal{K}}, \Sigma)>0$.

In other words, quadratic wavelet approximation on the boundary $\Sigma$ implies locally uniform approximation on $\Sigma_{\text {int }}$.

\section{Numerical examples}

Next, we present some sample examples for the geoscientifically important case of a sphere (i.e., $\Sigma$ is chosen to be the unit sphere $\Omega$ ).

\subsection{Spherical approximation of vector fields by layer potentials}

For this purpose, we first consider the vector field $f: \Omega \rightarrow \mathbb{R}^{3}$ given by

$$
f(x)= \begin{cases}0 \epsilon^{3}, & -1 \leq \xi \cdot \epsilon^{3} \leq h \\ \frac{3}{2}\left(\frac{h-\xi \cdot \epsilon^{3}}{h-1}\right)^{2} \epsilon^{3}, & h \leq \xi \cdot \epsilon^{3} \leq \frac{2+h}{3} \\ \left(1-3\left(\frac{\xi \cdot \epsilon^{3}-1}{h-1}\right)^{2}\right) \epsilon^{3}, & \frac{2+h}{3} \leq \xi \cdot \epsilon^{3} \leq 1\end{cases}
$$

for $x \in \Omega, \xi=x /|x|$, and $h=1 / 2$.

The third component of the boundary function $f$ is illustrated in Figure 6.1 . 


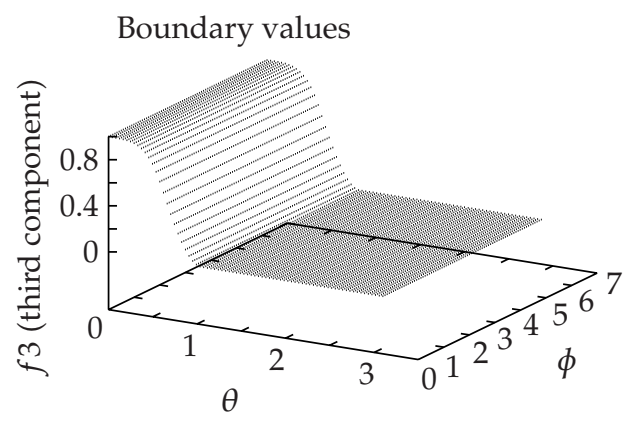

(a)

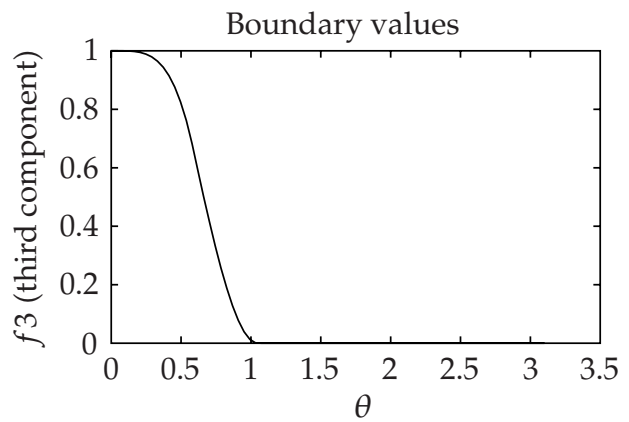

(b)

FIGURE 6.1. Functional values of $f$ (third component): (a) on a longitude-latitude grid of points on $\Omega$ and (b) one-dimensional sectional illustration.

We are particularly interested in approximating "the third component" of the vector function $f$ by our wavelet approach based on layer potentials (as prescribed above). Figure 6.2 shows the sectional illustration of approximations of the boundary function corresponding to the $\Omega$-scaling function $\mathbf{\Phi}_{\tau_{j}}^{(5)}$ for different levels, that is, $\tau_{j}=2^{-j}, j=1,2,3,4$. Note that, in each evaluation step, a sufficiently large number of equiangular longitude-latitude grid points on the unit sphere have been used in order to avoid oscillations in the approximation process.

In accordance with Theorem 4.1, it is clear that our multiscale procedure also provides a good approximation of $u \in \operatorname{pot}\left(\overline{\Omega_{\text {int }}}\right)$ with $\left.u\right|_{\Omega}=f$ inside $\Omega$. 
634 Multiscale deformation analysis by Cauchy-Navier wavelets

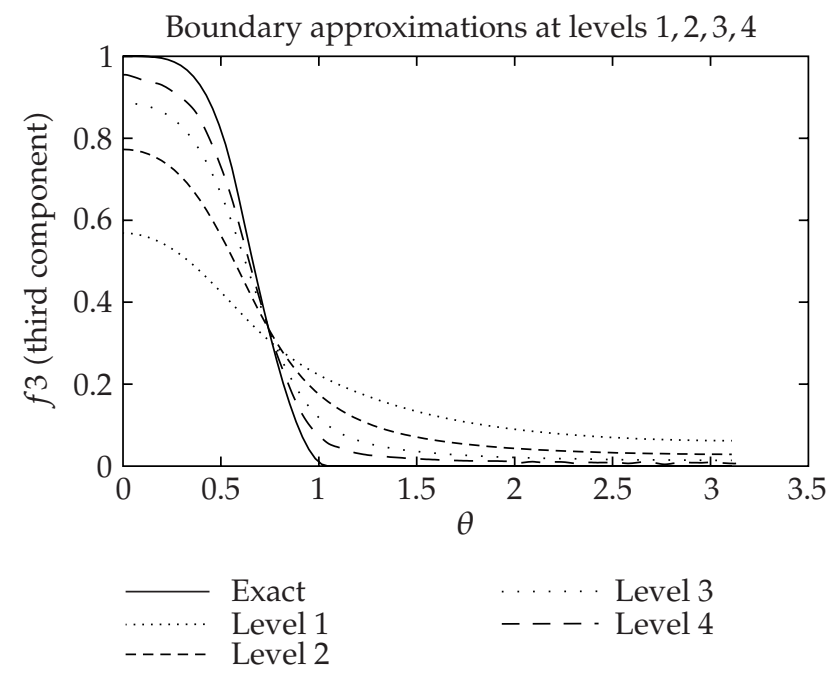

FigURE 6.2. Sectional illustration of the scale approximations of $f$

(third component) associated to the $\Omega$-scaling function $\boldsymbol{\Phi}_{\tau_{j}}^{(5)}$ for $j=1,2,3,4$.

\subsection{Solution of the displacement problem corresponding to a sphere}

Finally our multiscale method will be considered for the boundary approximation for a simple displacement boundary value problem of which the boundary is assumed to be the unit sphere $\Omega$ and an analytical solution is explicitly known in $\overline{\Omega_{\text {int }}}$. In contrast to the example discussed in Section 6.1 this one enables us to check the accuracy of the scale approximation in $\Omega_{\text {int }}$. However, we restrict ourselves to boundary approximation. The detail reconstructions are taken into account. To be more explicit, we consider the solution $u \in \operatorname{pot}\left(\overline{\Omega_{\text {int }}}\right)$ corresponding to the boundary field $\left.u\right|_{\Omega}=f$ given by

$$
f(x)=\frac{x_{1} x_{2}}{2} \epsilon^{1}-\frac{\sigma}{2(\sigma+3)}\left(x_{1}^{2}+x_{2}^{2}+x_{3}^{2}\right) \epsilon^{2}+\frac{x_{2} x_{3}}{2} \epsilon^{3}, \quad \sigma \neq 0 .
$$

We choose, in particular, $\sigma=2.5$ (i.e., $\lambda=2, \mu=3$ ). Figure 6.3 shows the exact boundary displacements under the displacement function $f$.

We compute the multiscale approximations of the boundary displacements on the sphere $\Omega$ for the first five levels. For the quantitative analysis of the accuracy, the radial and tangential displacements are taken into account. Figure 6.4 shows the exact radial displacement quantities by gray colormaps and tangential displacement vectors by arrows, respectively, on $\theta-\varphi$ polar coordinate plane. 


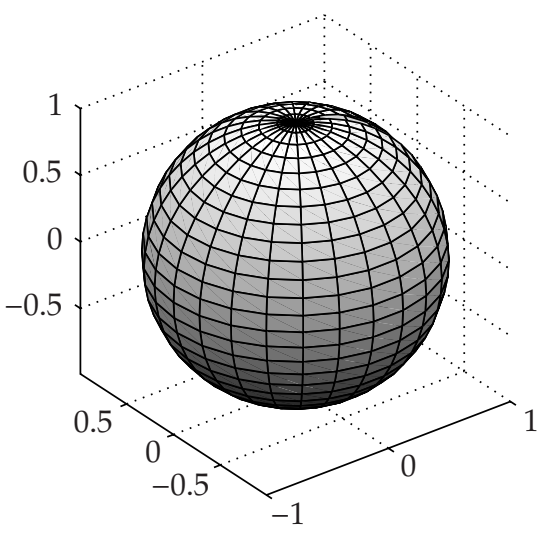

(a)

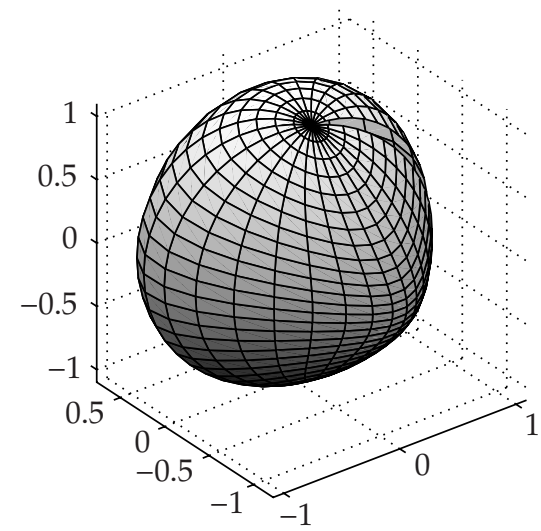

(b)

FIGURE 6.3. The reference (a) and the deformed (b) configurations of the unit sphere $\Omega$ associated to the displacement function $f$.

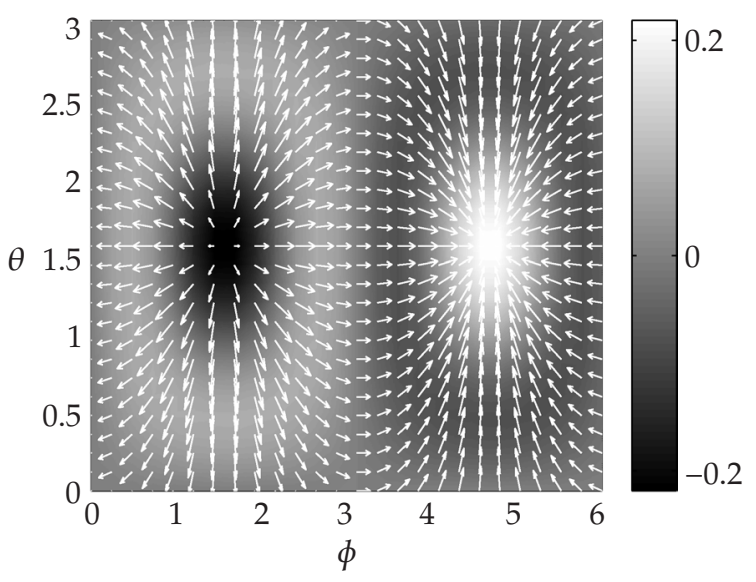

FIGURE 6.4. The (exact) radial and tangential boundary displacements on the unit sphere $\Omega$.

Since the evaluation of wavelet coefficients naturally consists of integrations over the unit sphere $\Omega$, we have to use an appropriate integration rule. In this respect, we use an equiangular longitude-latitude grid system where the integration weights are explicitly known (see, e.g., [4]). The following series of figures (Figures 6.5 and 6.6) show the corresponding multiscale approximations up to level 5 , in which we scale the size of arrows at each level taking the exact tangential displacement 
636 Multiscale deformation analysis by Cauchy-Navier wavelets

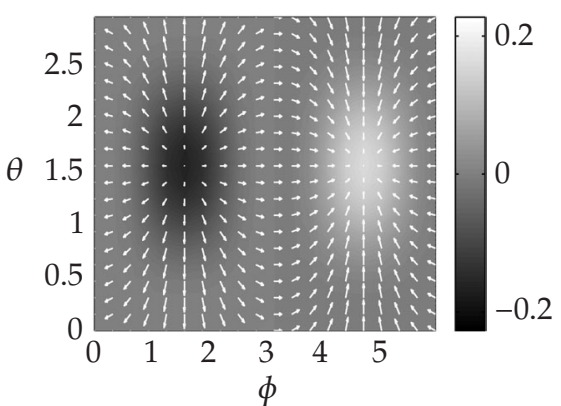

(a) Scale at $j=1$.

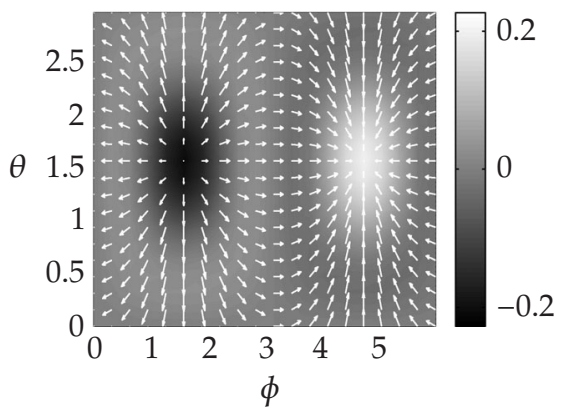

(c) Scale at $j=2$.

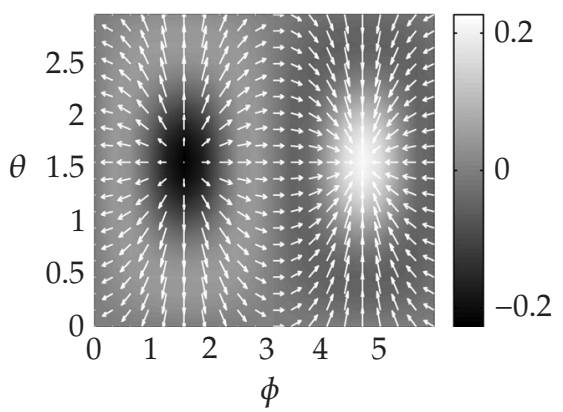

(e) Scale at $j=3$.

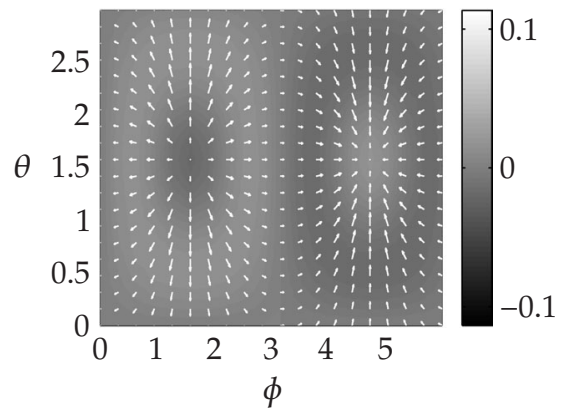

(b) Detail at $j=1$.

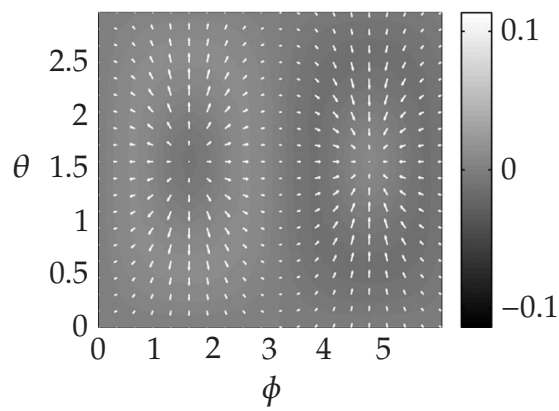

(d) Detail at $j=2$.

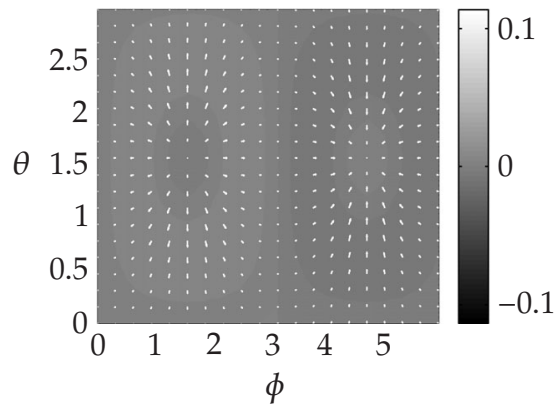

(f) Detail at $j=3$.

Figure 6.5. Scale (left) and detail (right) reconstructions at levels 1, 2 , and 3. 
M. K. Abeyratne et al. 637

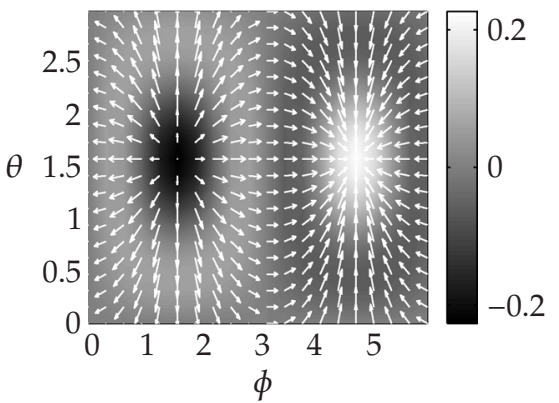

(a) Scale at $j=4$.

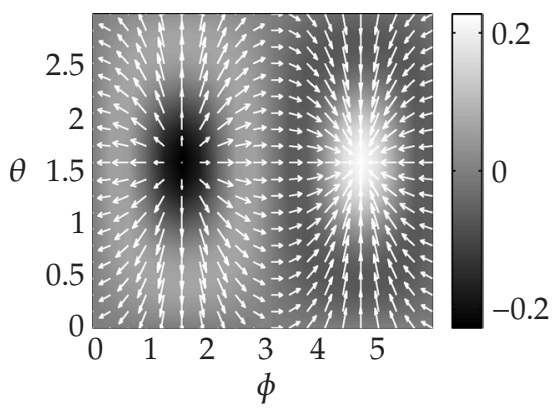

(c) Scale at $j=5$.

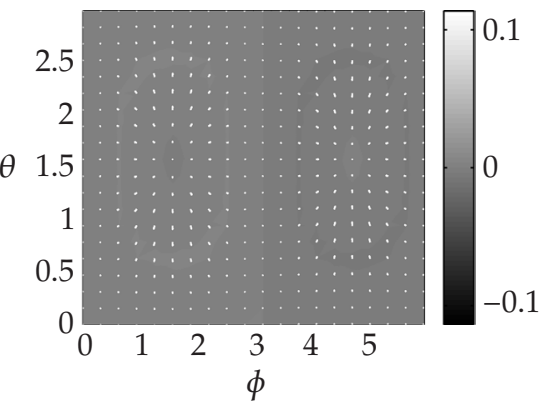

(b) Detail at $j=4$.

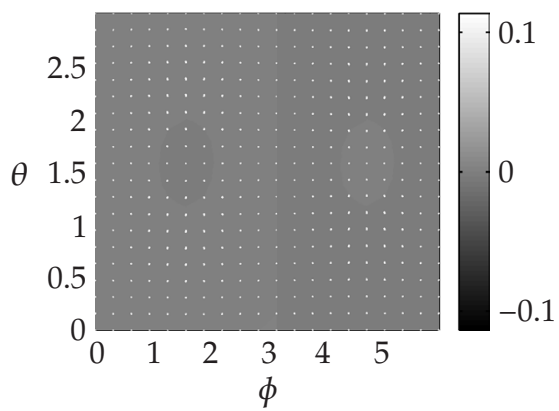

(d) Detail at $j=5$.

FIGURE 6.6. Scale (left) and detail (right) reconstructions at levels 4 and 5 .

vectors as the reference size. Moreover, to show the results in a more detailed form we rescale all detail information quantities multiplying by 2 . Furthermore, the same results are illustrated by Cartesian components in Figures 6.7, 6.8, 6.9, and 6.10.

More examples can be found in the Ph.D. thesis [1] of the first author.

\section{Concluding remarks}

In this paper we have introduced a multiscale method for solving the inner displacement problem using Cauchy-Navier wavelets. The method is particularly suitable for the deformation analysis corresponding to geoscientifically relevant boundaries (such as sphere, ellipsoid, actual earth surface, etc.) that involve efficient rules of numerical integration. 


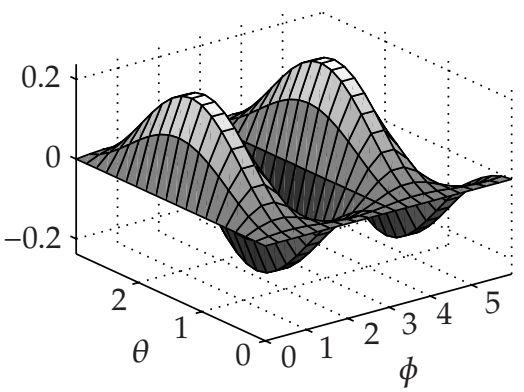

(a) Component $f \cdot \epsilon^{1}$.

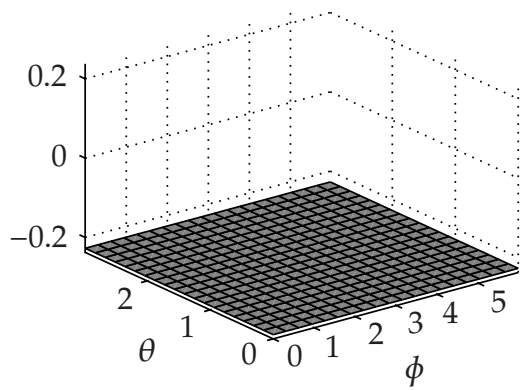

(b) Component $f \cdot \epsilon^{2}$.

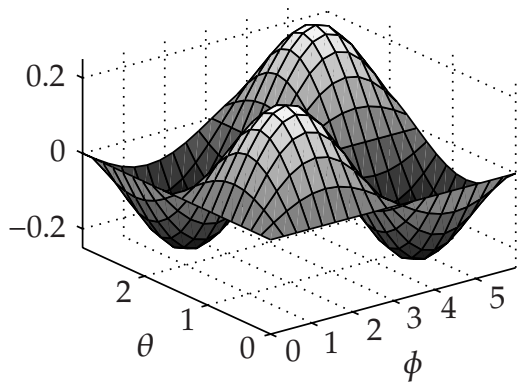

(c) Component $f \cdot \epsilon^{3}$.

FiguRE 6.7. Exact displacements in Cartesian components on $\Omega$.

The principal idea of the Cauchy-Navier wavelets is based on the classical limit and jump relations of elastostatics. In conclusion, this paper can be viewed as a first attempt to "short-wavelength modelling," that is, high resolution of the fine structure of displacement fields. The method is restricted to the homogeneous and isotropic case of linear elasticity, hence it needs to be formulated under more complex (geo-)physical assumptions.

Summarizing our results, we are led to the following conclusions: Cauchy-Navier wavelets are "vectorial building blocks" that enable fast modelling of displacement data. Hence, three features are incorporated in our way of thinking about Cauchy-Navier wavelets, namely, the basis property, decorrelation, and fast computation. By virtue of the basis property, each member of the Hilbert space $\ell^{2}(\Sigma)$ can be expressed as a linear combination involving products of dilated and shifted copies of 
M. K. Abeyratne et al. 639

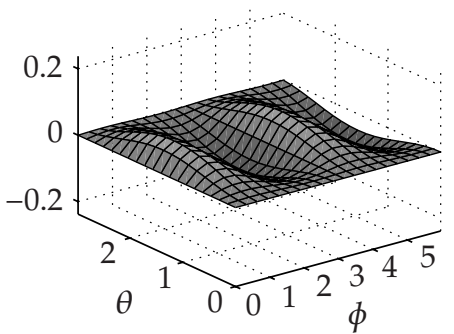

(a) Scale at $j=1$.

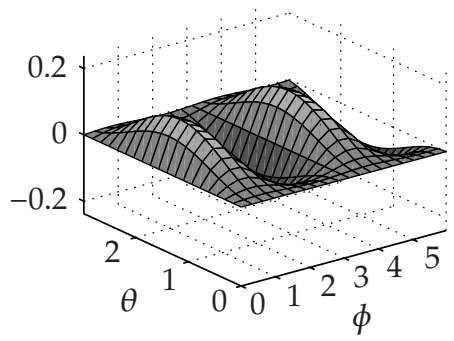

(c) Scale at $j=2$.

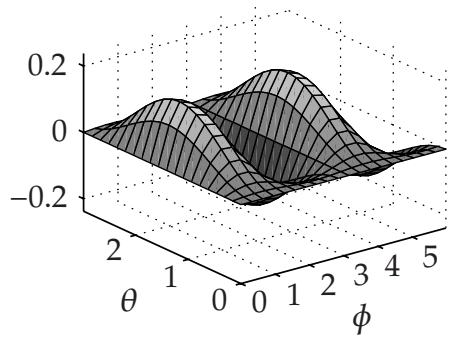

(e) Scale at $j=3$.

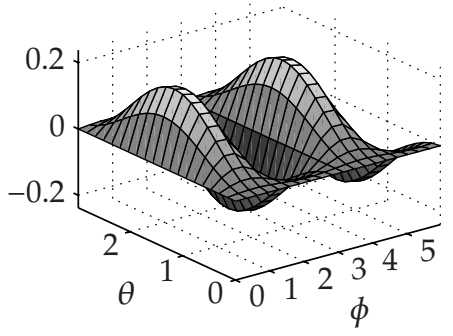

(g) Scale at $j=4$.

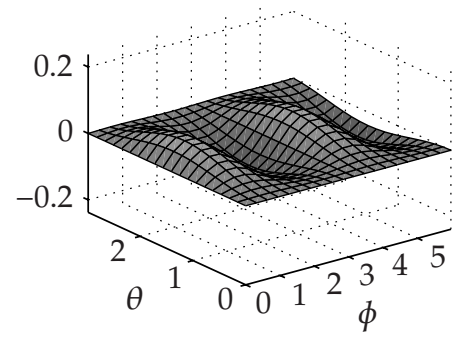

(b) Detail at $j=1$.

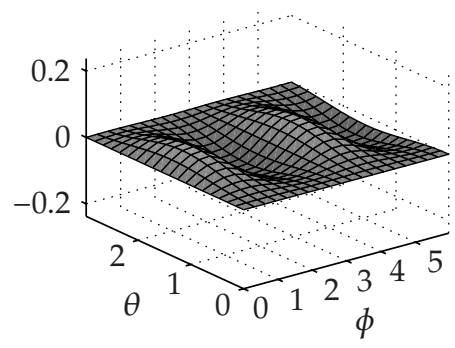

(d) Detail at $j=2$.

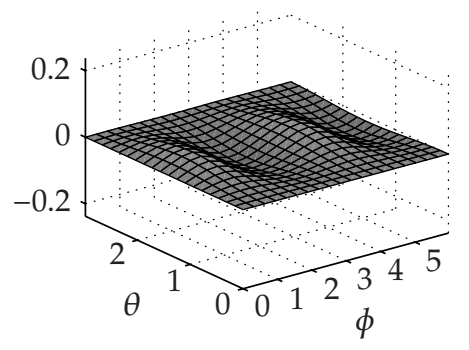

(f) Detail at $j=3$.

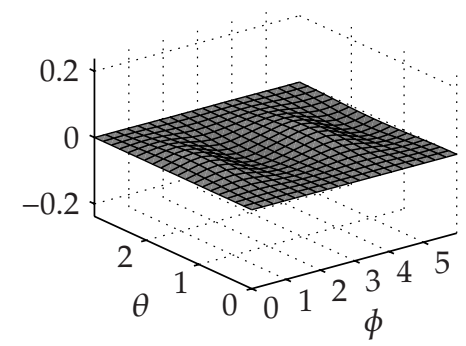

(h) Detail at $j=4$.

FIGURE 6.8. Scale (left) and detail (right) reconstructions ( $x_{1}$-component). 
640 Multiscale deformation analysis by Cauchy-Navier wavelets

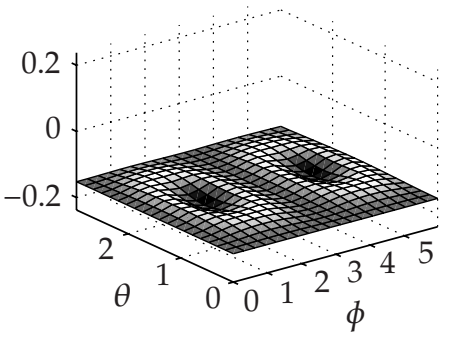

(a) Scale at $j=1$.

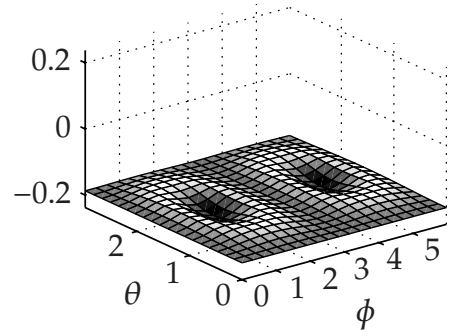

(c) Scale at $j=2$.

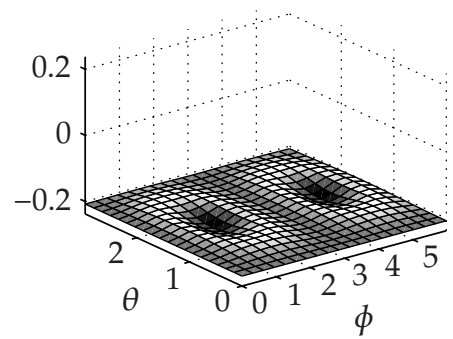

(e) Scale at $j=3$.

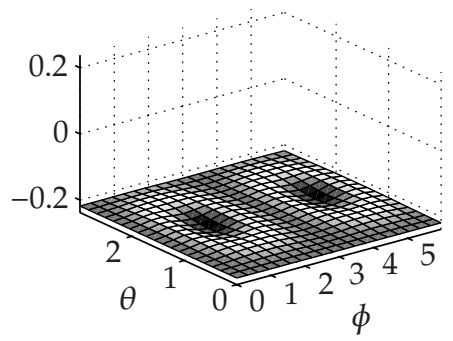

(g) Scale at $j=4$.

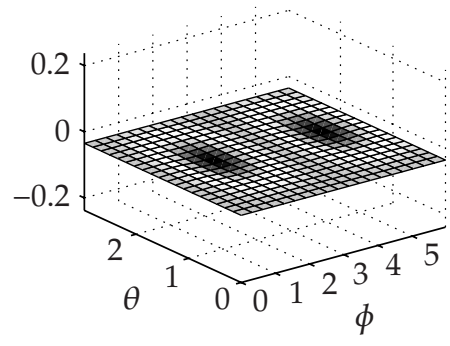

(b) Detail at $j=1$.

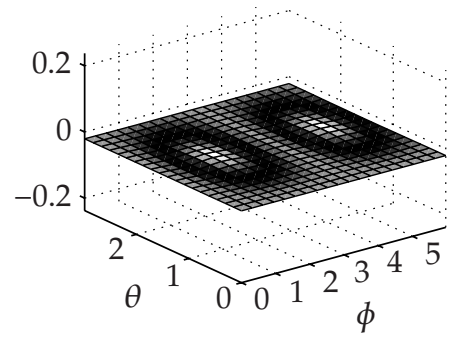

(d) Detail at $j=2$.

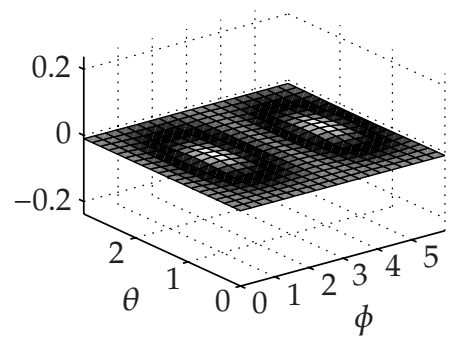

(f) Detail at $j=3$.

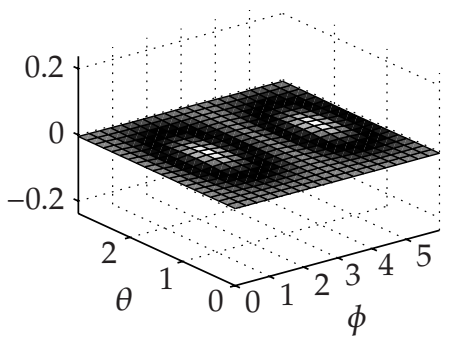

(h) Detail at $j=4$.

FIGURE 6.9. Scale (left) and detail (right) reconstructions ( $x_{2}$-component). 


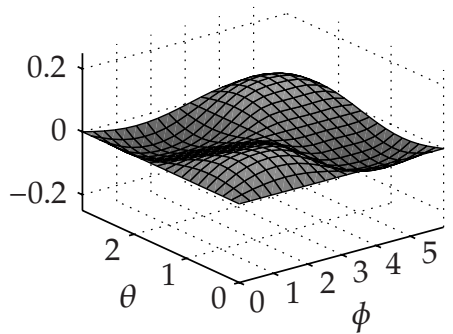

(a) Scale at $j=1$.

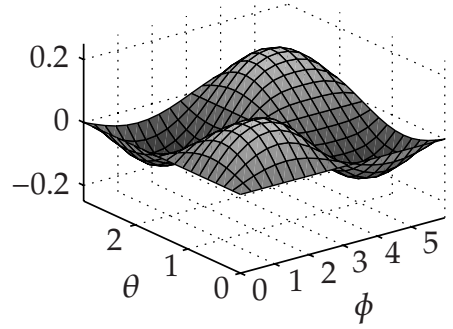

(c) Scale at $j=2$.

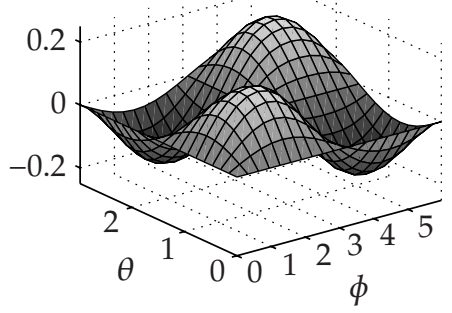

(e) Scale at $j=3$.

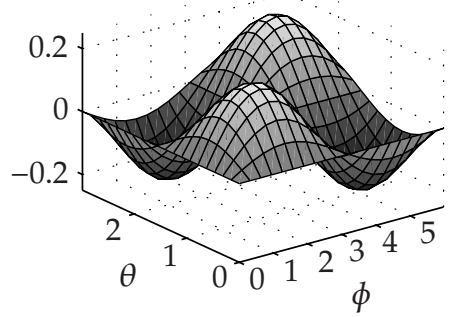

(g) Scale at $j=4$.

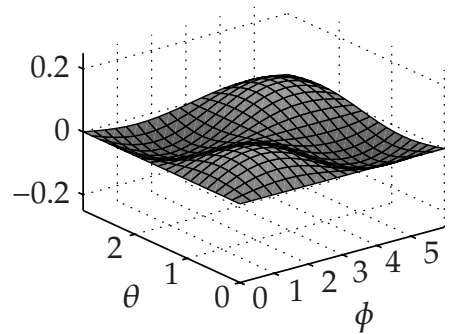

(b) Detail at $j=1$.

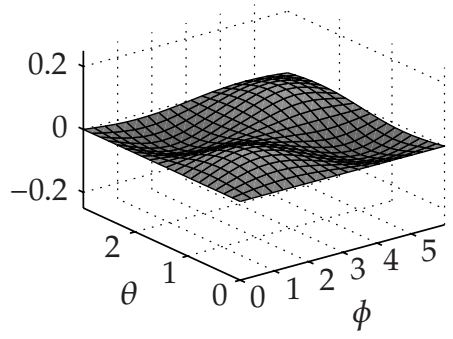

(d) Detail at $j=2$.

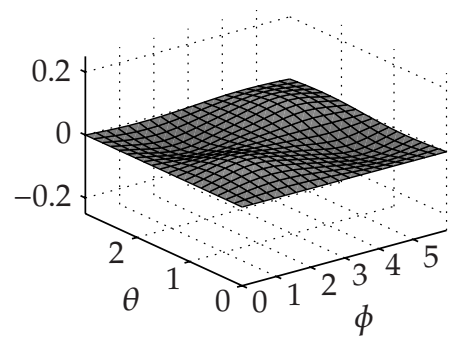

(f) Detail at $j=3$.

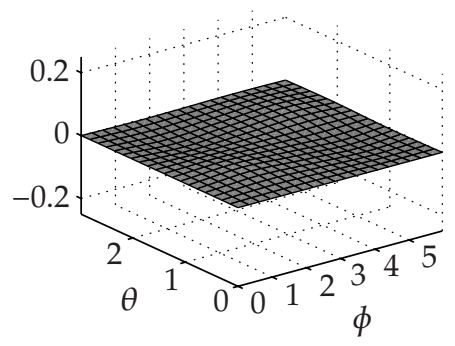

(h) Detail at $j=4$.

FIGURE 6.10. Scale (left) and detail (right) reconstructions ( $x_{3}$-components). 


\section{Multiscale deformation analysis by Cauchy-Navier wavelets}

a tensorial "mother kernel" with displacement data. The wavelet transform maps members of the Hilbert space $\ell^{2}(\Sigma)$ into an associated twoparameter class of scale- and space-dependent elements. Wavelets as constructed here show the power of decorrelation. As a consequence, the representation of displacement data is somehow "more compact" than the original representation, that is to say, wavelet modelling provides an accurate approximation by only using a small fraction of the original information. Typically, in the jargon of information theory, the convolutions $P_{j} f$ of tensorial scaling functions against the displacement field provide lowpass filtering at scale $j$, while the decorrelation is achieved by convolutions $R_{j} f$, that is, bandpass filtering. The essential questionalso seen in comparison to the other solution techniques for the displacement boundary value problem-is how to decompose a displacement field into wavelet coefficients and how to reconstruct efficiently the field from the wavelet coefficients. As a matter of fact, the investigations of our paper have led to a tree algorithm, that makes all steps simple and fast. Moreover, it is worth mentioning that the fast decorrelation power of Cauchy-Navier wavelets is the key to (future) applications such as data compression, fast data transmission, noise cancellation, and so forth.

Altogether, the multiscale procedure as presented here is an essential step for flexible and economical microstructural modelling of (isotropic homogeneous) elastic fields on (geoscientifically relevant) regular boundaries such as sphere, ellipsoid, or (actual) Earth surface.

\section{Appendix}

For the proof of Theorem 3.1 we use a modification of a technique due to [18]. Denote by $T(\tau)$ one of the operators $L_{i}^{ \pm}(\tau), i=1,2,3$, and $J_{i}(\tau), i=1,2,3,4,5$. Let $T^{*}(\tau)$ be the adjoint operator with respect to the inner product $(\cdot, \cdot) \ell^{2}(\Sigma)$. According to the Cauchy-Schwarz inequality we find

$$
\|T(\tau) g\|_{\ell^{2}(\Sigma)}^{2} \leq\|g\|_{\ell^{2}(\Sigma)}\left\|T^{*}(\tau) T(\tau)\right\|_{\ell^{2}(\Sigma)} .
$$

Therefore it follows that

$$
\begin{aligned}
\|T(\tau) g\|_{\ell^{2}(\Sigma)}^{2^{2}} & \leq\|g\|_{\ell^{2}(\Sigma)}^{2}\left\|T^{*}(\tau) T(\tau)\right\|_{\ell^{2}(\Sigma)}^{2} \\
& \leq\|g\|_{\ell^{2}(\Sigma)}^{2}\|g\|_{\ell^{2}(\Sigma)}\left\|\left(T^{*}(\tau) T(\tau)\right)^{2} g\right\|_{\ell^{2}(\Sigma)}^{2} .
\end{aligned}
$$

Induction states that for all $n \geq 2$,

$$
\|T(\tau) g\|_{\ell^{2}(\Sigma)}^{2^{n}} \leq\|g\|_{\ell^{2}(\Sigma)}^{2^{n}-1}\left\|\left(T^{*}(\tau) T(\tau)\right)^{2^{n-1}} g\right\|_{\ell^{2}(\Sigma)} .
$$


Because of the boundedness of the operators $T^{*}(\tau)$ and $T(\tau)$ with respect to $\|\cdot\|_{c(\Sigma)}$ there exists a positive constant $D$ such that

$$
\|T(\tau) g\|_{\ell^{2}(\Sigma)}^{2^{n}} \leq\|\Sigma\| D^{2^{n}}\|g\|_{\ell^{2}(\Sigma)}^{2^{n}-1}\|g\|_{c(\Sigma)}, \quad\|\Sigma\|=\left(\int_{\Sigma} d \omega\right)^{1 / 2} .
$$

Thus, for all $n \geq 2$ and $g \in c(\Sigma)$ with $g \neq 0$, we obtain

$$
\frac{\|T(\tau) g\|_{\ell^{2}(\Sigma)}}{\|g\|_{\ell^{2}(\Sigma)}} \leq D\left(\frac{\|\Sigma\|\|g\|_{c(\Sigma)}}{\|g\|_{\ell^{2}(\Sigma)}}\right)^{2^{-n}} .
$$

Letting $n$ tend to infinity, we obtain for all $g \neq 0$

$$
\lim _{n \rightarrow \infty}\left(\frac{\|g\|_{c(\Sigma)}}{\|g\|_{\ell^{2}(\Sigma)}}\right)^{2^{-n}}=1
$$

This shows that $\|T(\tau)\|_{\ell^{2}(\Sigma)} \leq D$ for all $g \in c(\Sigma)$. Since $c(\Sigma)$ is a dense linear subspace of $\ell^{2}(\Sigma)$, we are able to extend the operator $T(\tau)$ from $c(\Sigma)$ to $\ell^{2}(\Sigma)$ without enlarging its norm (cf. $\left.[2,8,19]\right)$. Therefore, $T(\tau)$ is bounded with respect to $\|\cdot\|_{\ell^{2}(\Sigma)}$ and we have

$$
\|T(\tau)\|_{\ell^{2}(\Sigma)} \leq \sqrt{\|T(\tau)\|_{c(\Sigma)}\left\|T^{*}(\tau)\right\|_{c(\Sigma)}} .
$$

Hence it follows that $\|T(\tau)\|_{\ell^{2}(\Sigma)} \rightarrow 0$ as $\tau \rightarrow 0, \tau>0$.

\section{Acknowledgments}

This work is supported by the German Academic Exchange Service (DAAD) and the Graduate Research Training Programme (Graduiertenkolleg) "Mathematik und Praxis," sponsored by the German Research Foundation (DFG).

\section{References}

[1] M. K. Abeyratne, Cauchy-Navier wavelet solvers and their application in deformation analysis, Ph.D. thesis, Geomathematics Group, Department of Mathematics, University of Kaiserslautern, Kaiserslautern, 2003.

[2] N. I. Achieser and I. M. Glasmann, Theorie der linearen Operatoren im HilbertRaum [Theory of Linear Operators in Hilbert Space], Verlag Harri Deutsch, Thun, 1981.

[3] H. Bauch, Approximationssätze für die Lösungen der Grundgleichung der Elastostatik, Ph.D. thesis, Institut für Reine und Angewandte Mathematik, RWTH Aachen, Aachen, 1981. 


\section{Multiscale deformation analysis by Cauchy-Navier wavelets}

[4] J. R. Driscoll and D. M. Healy Jr., Computing Fourier transforms and convolutions on the 2-sphere, Adv. in Appl. Math. 15 (1994), no. 2, 202-250.

[5] W. Freeden, Least squares approximation by linear combinations of (multi-)poles, Tech. Report 344, Department of Geodetic Science and Surveying, The Ohio State University, Ohio, 1983.

[6]___ Multiscale Modelling of Spaceborne Geodata, European Consortium for Mathematics in Industry, B. G. Teubner, Stuttgart, 1999.

[7] W. Freeden, T. Gervens, and M. Schreiner, Constructive Approximation on the Sphere with Applications to Geomathematics, Numerical Mathematics and Scientific Computation, Clarendon Press, Oxford University Press, New York, 1998.

[8] W. Freeden and H. Kersten, A constructive approximation theorem for the oblique derivative problem in potential theory, Math. Methods Appl. Sci. 3 (1981), no. $1,104-114$.

[9] W. Freeden and R. Reuter, A constructive method for solving the displacement boundary-value problem of elastostatics by use of global basis systems, Math. Methods Appl. Sci. 12 (1990), no. 2, 105-128.

[10] W. Freeden and U. Windheuser, Spherical wavelet transform and its discretization, Adv. Comput. Math. 5 (1996), no. 1, 51-94.

[11] O. Glockner, On numerical aspects of gravitational field modelling from SST and SGG by harmonic splines and wavelets (with application to CHAMP data), Ph.D. thesis, Geomathematics Group, Department of Mathematics, University of Kaiserslautern, Kaiserslautern, 2002.

[12] M. E. Gurtin, Theory of Elasticity, 2nd ed., Handbuch der Physik, vol. 6, Springer-Verlag, Berlin, 1991.

[13] W. Hackbusch and Z. P. Nowak, On the fast matrix multiplication in the boundary element method by panel clustering, Numer. Math. 54 (1989), no. 4, 463491.

[14] K. Hesse, Domain decomposition methods in multiscale geopotential determination from SST and SGG, Ph.D. thesis, Geomathematics Group, Department of Mathematics, University of Kaiserslautern, Kaiserslautern, 2003.

[15] M. A. Jawson and G. T. Symm, Integral Equation Methods in Potential Theory and Elastostatics, Computational Mathematics and Applications, Academic Press, New York, 1977.

[16] R. J. Knops and L. E. Payne, Uniqueness Theorems in Linear Elasticity, SpringerVerlag, New York, 1971.

[17] V. D. Kupradze, Potential Methods in the Theory of Elasticity, Israel Program for Scientific Translations, Jerusalem, 1965.

[18] P. D. Lax, Symmetrizable linear transformations, Comm. Pure Appl. Math. 7 (1954), 633-647.

[19] L. A. Ljusternik and W. I. Sobolew, Elemente der Funktionalanalysis, Akademie-Verlag, Berlin, 1955.

[20] A. I. Lurje, Räumliche Probleme der Elastizitätstheorie, Akademie-Verlag, Berlin, 1963.

[21] J. E. Marsden and T. J. R. Hughes, Mathematical Foundations of Elasticity, Dover Publications, New York, 1994.

[22] I. S. Sokolnikoff, Mathematical Theory of Elasticity, 2nd ed., McGraw-Hill, New York, 1956.

[23] K. Yoshida, Functional Analysis, Springer-Verlag, New York, 1965. 
M. K. Abeyratne: Geomathematics Group, Department of Mathematics, University of Kaiserslautern, P.O. Box 3049, 67653 Kaiserslautern, Germany

E-mail address: abeyratn@mathematik.uni-kl.de

W. Freeden: Geomathematics Group, University of Kaiserslautern, Department of Mathematics, P.O. Box 3049, 67653 Kaiserslautern, Germany

E-mail address: freeden@mathematik.uni-kl.de

C. Mayer: Geomathematics Group, University of Kaiserslautern, Department of Mathematics, P.O. Box 3049, 67653 Kaiserslautern, Germany

E-mail address: cmayer@mathematik.uni-kl.de 


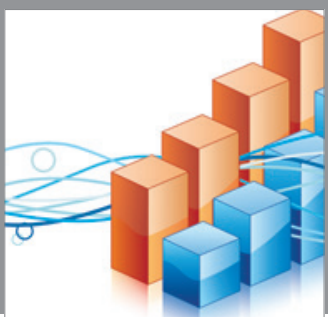

Advances in

Operations Research

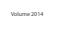

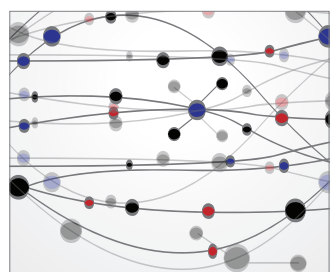

\section{The Scientific} World Journal
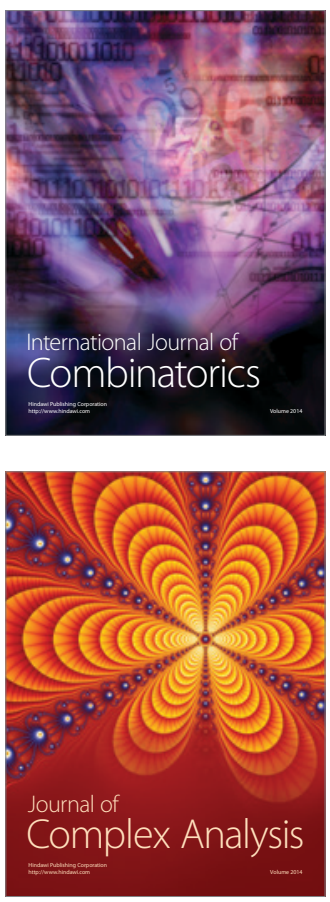

International Journal of

Mathematics and

Mathematical

Sciences
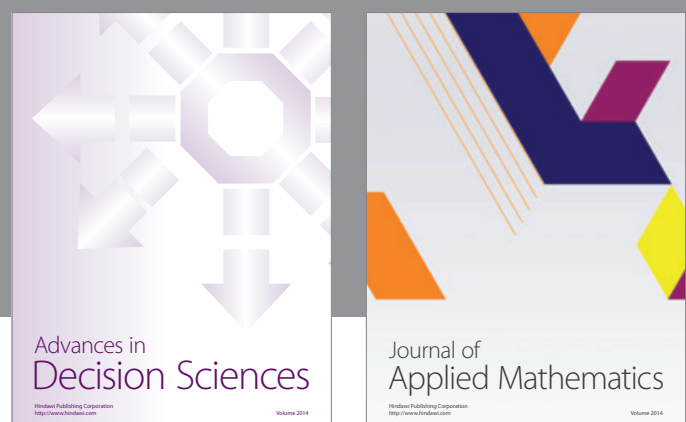

Journal of

Applied Mathematics
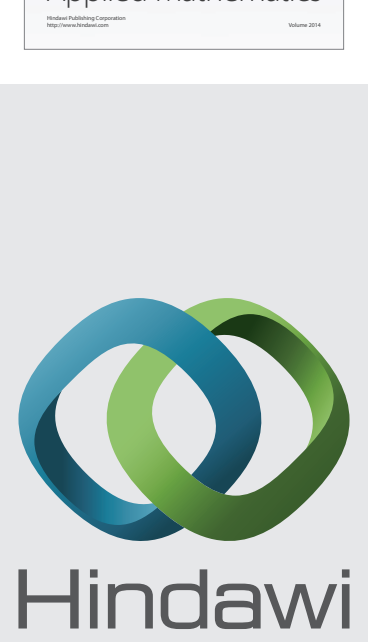

Submit your manuscripts at http://www.hindawi.com
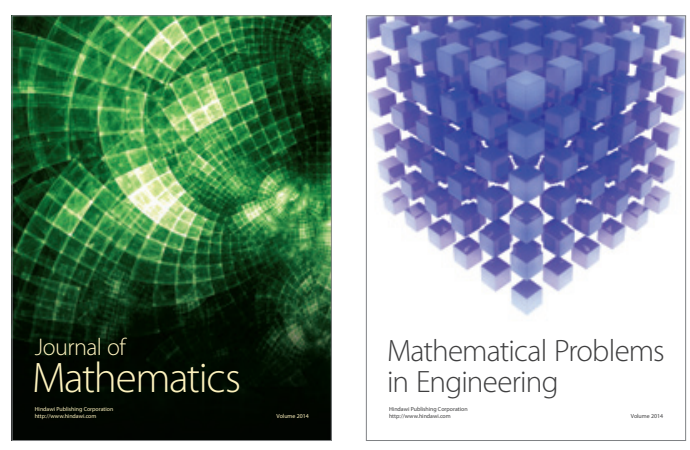

Mathematical Problems in Engineering
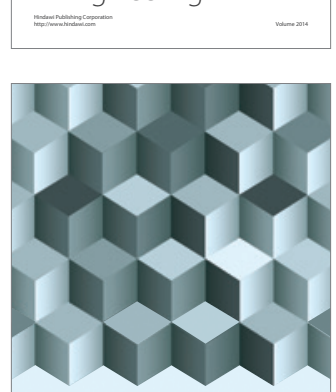

Journal of

Function Spaces
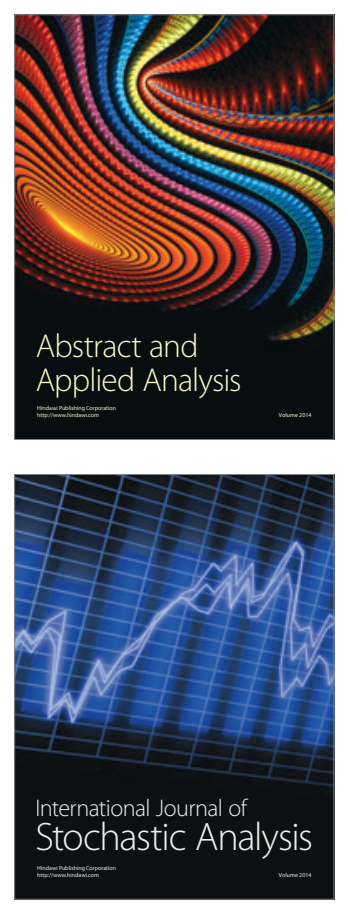

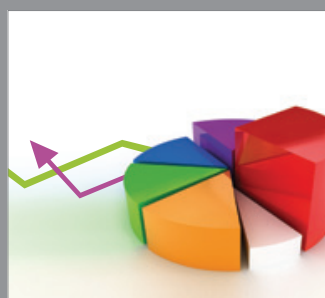

ournal of

Probability and Statistics

Promensencen
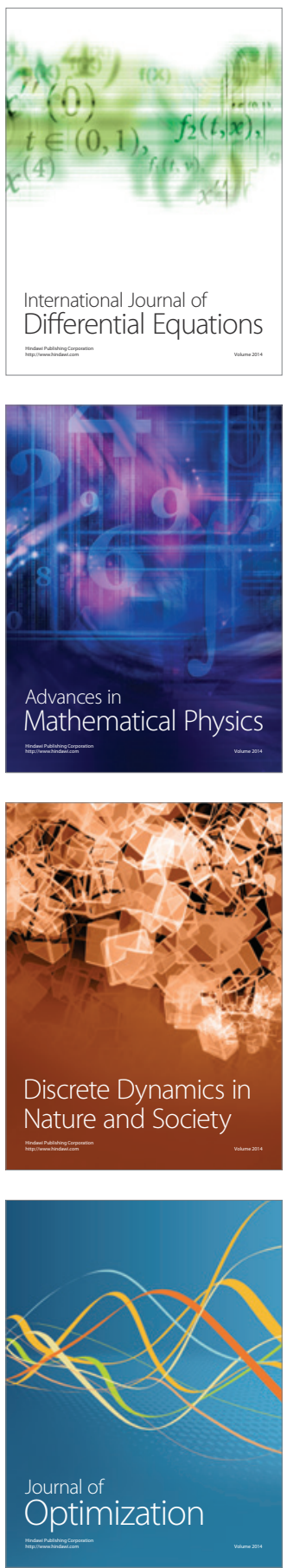